\title{
A local-influence-based diagnostic approach to a speeded item response theory model
}

\author{
Yuri Goegebeur, \\ Katholieke Universiteit Leuven, Belgium, and University of Southern Denmark, Odense, \\ Denmark
}

Paul De Boeck,

Katholieke Universiteit Leuven, Belgium

Geert Molenberghs

Hasselt University, Diepenbeek, Belgium

and Guido del Pino

Pontificia Universidad Católica de Chile, Santiago, Chile

[Received November 2005. Final revision May 2006]

\begin{abstract}
Summary. An item response theory model for dealing with omitted responses in a test is proposed. In this model formulation, non-response does not only depend on an examinee's ability and on item difficulty, but additionally also on 'test speededness'. Using a local-influence-based diagnostic approach, the sensitivity of the model regarding assumptions concerning the dropout mechanism is explored. The methodology proposed is applied to the Chilean Sistema de Medición de la Calidad de la Educación mathematics test case-study.
\end{abstract}

Keywords: Item response theory; Local influence; Missing data; 'Test speededness'

\section{Introduction}

The Sistema de Medición de la Calidad de la Educación project in Chile (known as 'SIMCE') has developed compulsory language and mathematics tests to assess regularly the educational progress in three levels: fourth, eighth and 10th graders. All students in the grade level in the country (public, private and mixed support schools) are expected to take the tests whenever scheduled (every 3-4 years). The number of examinees can be as large as 200000 per test. The items are polytomous and the students were asked to respond to all of them. However, about $20 \%$ of the response profiles contained at least one missing value. The results of the SIMCE tests are relevant for the schools and the Government but do not have individual consequences for the students.

The purpose of this paper is

(a) to develop a possible explanation for the non-response in test data and

(b) to assess the effect of the model assumptions, in particular those which are related to the posited non-response mechanism, on the statistical conclusions.

Address for correspondence: Yuri Goegebeur, Department of Statistics, University of Southern Denmark, J. B. Winsløws Vej 9B, DK-5000 Odense C, Denmark.

E-mail: yuri.goegebeur@stat.sdu.dk 
The first objective is pursued by considering non-response as emerging from a tendency to omit an item in case one does not know the answer and from an effect standardly referred to as 'test speededness' (i.e. an adverse effect due to the limited time test administration; see for instance Bolt et al. (2002)), both taken to be examinee specific. Compared with a complete-case analysis, this approach allows the inclusion of incomplete response profiles and hence offers opportunities for improved parameter estimation. The second objective is addressed by a formal sensitivity analysis of the non-response mechanism. To be precise, we aim to identify examinees who do not fit a particular postulated model because their response profiles are affected by test speededness effects. This approach allows us to examine the magnitude of the support for a particular model in case we must choose between two competing models.

We shall consider the data from the 2001 administration of the SIMCE mathematics test to the 10th graders in public schools. The mathematics test contains 48 items, each having four response alternatives, and covers topics such as problem formulation, functions, simple algebra, geometry and probability. For instance, students are asked to simplify

$$
\frac{4}{x^{2}} / \frac{2}{x}
$$

or to compute $30 \%$ of $\$ 2000$ in the context of an applied problem. The test is administered under a fixed time limit of $90 \mathrm{~min}$. The database under consideration contains response profiles of 36118 examinees. The methodology that is proposed in this paper is applied to a sample of 3000 examinees who are randomly drawn from this database. In Fig. 1, the sample is summarized by plotting the proportions of omitted answers (full curve), incorrect answers (broken curve) and correct answers (chain curve) as a function of the item number. The proportions of omitted answers vary between 0.0020 and 0.0537 with mean 0.0176 and standard deviation 0.0117 . Of the 3000 examinees, $626(20.87 \%)$ have a response profile with at least one answer omitted. Although the SIMCE test is not used for decisions at the individual level, this is quite a large percentage. In other applications, the individual level may be important for decisions, and the present study may have interesting implications for such other applications as well. Note also that the proportion of items omitted slightly increases with the item number, which is an effect that may be due to the fixed time limit administration of the test. The proportions of incorrect answers are in the range [0.1600,0.7889] with mean 0.4745 and standard deviation 0.1589 . Finally, the proportions of correct answers are between 0.1913 and 0.8380 with mean 0.5079 and standard deviation 0.1660 .

We shall derive an item response theory (IRT) model accommodating non-response. The missing observations are modelled simultaneously with the observed responses, by treating the data as if there were three response categories: correct, incorrect and no response. Moreover, the model accommodates the possible adverse effects of limited time test administrations. As this model builds on classical IRT models and, incidentally, also fits within the missing data framework that was proposed by Rubin (1976) and Little and Rubin (2002), it is instructive to review some of these concepts.

Let $Y_{p i}$ denote the binary response (correct-incorrect, coded $Y_{p i}=1$ and $Y_{p i}=0$ respectively) of examinee $p, p=1, \ldots, P$, toitem $i, i=1, \ldots, I$.In theclassical one-parameter Rasch model(Rasch, 1960) $Y_{p i}$ depends on the examinee's ability $\theta_{p}$ and the item difficulty $\beta_{i}$ in the following way:

$$
Y_{p i} \mid \theta_{p} \sim \operatorname{Bern}\left\{P_{i}\left(\theta_{p}\right)\right\}
$$

with

$$
P_{i}\left(\theta_{p}\right)=\frac{\exp \left(\theta_{p}-\beta_{i}\right)}{1+\exp \left(\theta_{p}-\beta_{i}\right)},
$$




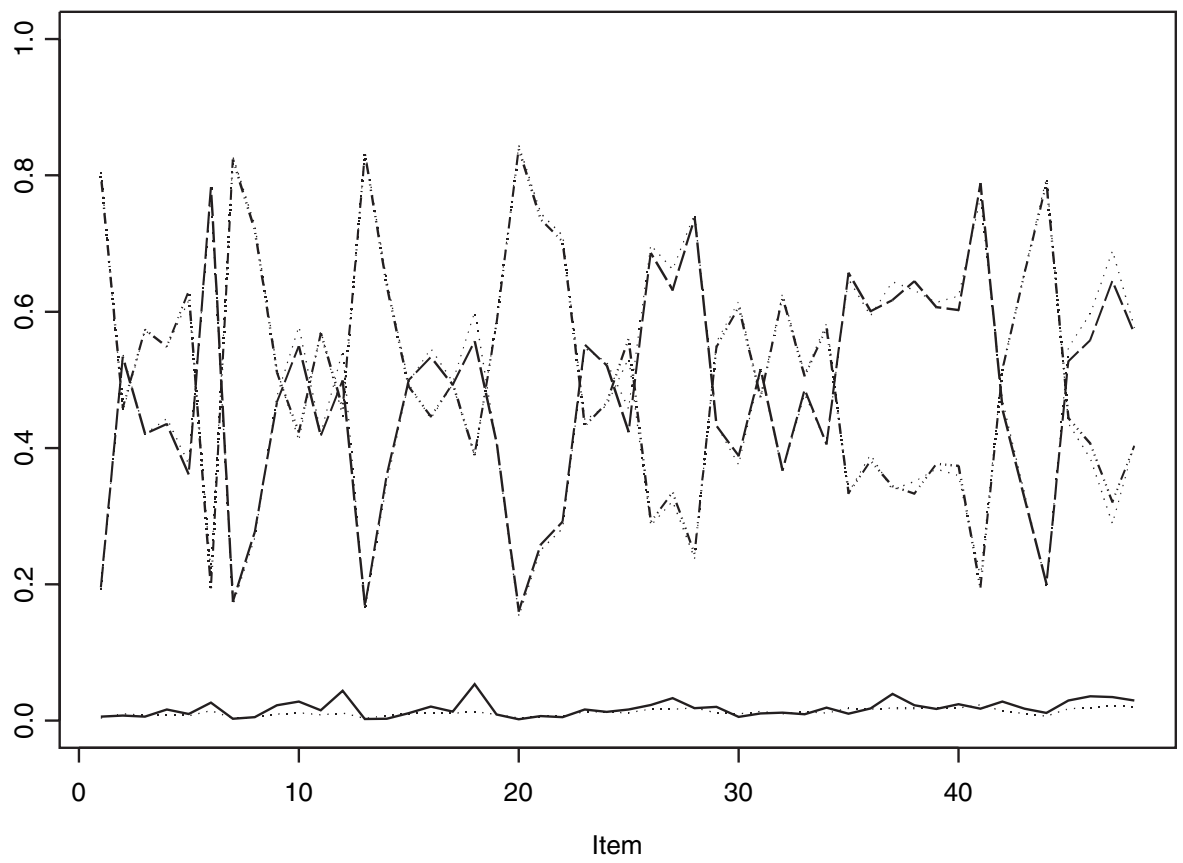

Fig. 1. Proportion of missing data ( - ), incorrect answers ( - ) and correct answers $(\cdots \cdots \cdots)$ together with the estimated theoretical proportion under the test model $(\cdots \cdots)$

and $\theta_{p} \sim N\left(0, \sigma_{\theta}^{2}\right)$. Moreover, conditional on $\theta_{p}$, all responses of subject $p$ are assumed to be independent: the so-called local item independence condition. The Rasch model has been extended in several ways. In the two-parameter logistic model (Birnbaum, 1968) the random intercept is weighted by an item parameter $\alpha_{i}$, called the discrimination parameter:

$$
P_{i}\left(\theta_{p}\right)=\frac{\exp \left(\alpha_{i} \theta_{p}-\beta_{i}\right)}{1+\exp \left(\alpha_{i} \theta_{p}-\beta_{i}\right)},
$$

so that the influence of the examinee's ability on outcome depends on the item. The three-parameter logistic model (Birnbaum, 1968) extends the two-parameter model with an itemspecific guessing parameter $c_{i}$ :

$$
P_{i}\left(\theta_{p}\right)=c_{i}+\left(1-c_{i}\right) \frac{\exp \left(\alpha_{i} \theta_{p}-\beta_{i}\right)}{1+\exp \left(\alpha_{i} \theta_{p}-\beta_{i}\right)} .
$$

The guessing parameter $c_{i}$ represents the probability of a correct answer under random guessing. San Martín et al. (2006) further extended this model by considering the possibility that the examinee uses his or her ability to some extent to differentiate between responses in the process of guessing.

IRT models in general and the Rasch model in particular are not robust against violations of the local item independence assumption. The inclusion of items with local item dependence may result in contaminated estimates of test reliability, person and item parameters, standard errors and equating coefficients. This has been reported in Yen (1984), Thissen et al. (1989), Sireci et al. (1991), Yen (1993), Wainer and Thissen (1996), Lee et al. (2001) and Tuerlinckx and De Boeck (2001).

Yen (1993) and Ferrara et al. (1999) have provided a detailed taxonomy of possible reasons for the existence of local item dependence. One of the most prevalent causes in educational 
testing is test speededness, which refers to testing situations in which some examinees do not have ample time to answer all questions. These effects are often detrimental to the intended functioning of the test in the sense that the speed with which one responds is usually not an important part of the construct of interest, yet examinees who are affected by it hurry through, randomly guess on or even fail to complete items, usually at the end of the test, and hence receive estimates of ability that underestimate their abilities. Moreover, the item difficulty parameters of items administered late in the test tend to be overestimated (Douglas et al., 1998; Oshima, 1994). Item response models accommodating test speededness were proposed by Yamamoto and Everson (1997), Bolt et al. (2002), Wollack and Cohen (2005) and Goegebeur et al. (2005). Although these models provide improved parameter estimates, they do not explicitly address the issue of omitted responses. Nevertheless, such omitted responses can be informative in the sense that they can provide information about the ability of examinees, the difficulty of items and possibly test speededness.

Rubin (1976) and Little and Rubin (2002), chapter 6, established a framework to distinguish between different missing values processes. A missing value process is said to be missing completely at random if missingness is independent of both observed and unobserved data and missing at random if, conditional on the observed data, missingness does not depend on the unobserved data; otherwise the missingness process is termed missingness not at random (MNAR). If the missingness process is random and the parameters of the observation process are functionally independent of the parameters describing the missingness process, then a valid statistical analysis can be obtained through a likelihood-based analysis (or a Bayesian analysis) that ignores the missigness mechanism. This situation was termed ignorable by Rubin (1976) and Little and Rubin (2002).

Although historically most methods were framed in the missingness completely at random category, for computational and other simplicity reasons, more work has been done in the missingness at random and more recently in the MNAR category (see for instance Little (1995), Hogan and Laird (1997), Schafer (1997), Verbeke and Molenberghs (2000), Little and Rubin (2002), Molenberghs and Verbeke (2005) and the references therein). In many testing situations, including our context, missingness often depends on latent data such as ability of the examinees and sensitivity to test speededness. This would point to MNAR, which is non-ignorable, regardless of the inferential mode that is chosen. Many researchers have warned against too firm a belief in a single (MNAR) model since, owing to the very nature of incompleteness, such a model cannot be verified from observed data only. This implies great sensitivity to model assumptions (Verbeke and Molenberghs, 2000; Molenberghs and Verbeke, 2005). These issues are compounded when, in addition to incomplete data, the models feature latent structure, (unobserved) random effects, etc. We are in need of a model that combines all these. Apart from random guessing, random subject effects and test speededness, incompleteness occurs and there are probably interrelationships between these entities. Therefore, model formulation should go hand in hand with so-called sensitivity analysis. In particular, we shall follow the local influence route, which was applied by Lesaffre and Verbeke (1998) to the linear mixed model and by Verbeke et al. (2001) to the linear mixed model with MNAR. The approach will allow us to identify and study subjects further that have a strong effect on the key model parameters.

The remainder of this paper is organized as follows. In Section 2 we propose a model for omitted responses also incorporating test speededness effects. This model is derived from a decision tree that describes the examinee's possible states and actions when she or he encounters an item. In Section 3 we focus on the use of global and local influence diagnostics as tools to examine the sensitivity of the estimation results with respect to aberrant response patterns. In particular we concentrate on the normal curvature measures that were proposed by Cook (1986) as these 
allow us to identify response profiles that do not fit a particular aspect of the model. Throughout the paper the methodology proposed is applied to the SIMCE mathematics test data.

The programs that were used to analyse the date can be obtained from

http: / /www.blackwellpublishing.com/rss

\section{A model for test speededness and omitted responses}

When subject $p$ encounters item $i$ she or he is either knowledgeable or ignorant. If knowledgeable, the probability of a correct answer, denoted $P_{i}\left(\theta_{p}\right)$, is given by equation (1) or (2). If ignorant, the examinee omits the item with probability $P_{i}\left(\xi_{0 p}, \xi_{1 p}\right)$ and guesses at random with probability $1-P_{i}\left(\xi_{0 p}, \xi_{1 p}\right)$, where we assume that

$$
P_{i}\left(\xi_{0 p}, \xi_{1 p}\right)=\frac{\exp \left(\xi_{0 p}+\xi_{1 p} i / I\right)}{1+\exp \left(\xi_{0 p}+\xi_{1 p} i / I\right)}, \quad \xi_{0 p} \in \mathbb{R}, \quad \xi_{1 p}>0 .
$$

The random effect $\xi_{0 p}$ can be seen as the initial propensity of examinee $p$ to omit items, whereas $\xi_{1 p}$ reflects the examinee-specific effect of test speededness, where speeding increases the probability of an omitted response. In the case that the examinee guesses at random, the answer is correct with probability $c$. In Fig. 2 the process that is described above is visually represented by a decision tree.

Clearly, this decision tree involves a categorical response variable with three possible levels: no answer, wrong answer and correct answer, coded $\mathbf{Y}_{p i}^{\prime}:=\left(Y_{p i 0}, Y_{p i 1}\right)=(1,0), \mathbf{Y}_{p i}^{\prime}=(0,1)$ and $\mathbf{Y}_{p i}^{\prime}=(0,0)$ respectively. The corresponding conditional probabilities will be denoted by $\pi_{p i 0}$, $\pi_{p i 1}$ and $\pi_{p i 2}$ respectively and have expressions that follow immediately from Fig. 2:

$$
\begin{gathered}
\pi_{p i 0}=\left\{1-P_{i}\left(\theta_{p}\right)\right\} P_{i}\left(\xi_{0 p}, \xi_{1 p}\right), \\
\pi_{p i 1}=\left\{1-P_{i}\left(\theta_{p}\right)\right\}\left\{1-P_{i}\left(\xi_{0 p}, \xi_{1 p}\right)\right\}(1-c), \\
\pi_{p i 2}=\left\{1-P_{i}\left(\xi_{0 p}, \xi_{1 p}\right)\right\} c+\left[1-\left\{1-P_{i}\left(\xi_{0 p}, \xi_{1 p}\right)\right\} c\right] P_{i}\left(\theta_{p}\right) .
\end{gathered}
$$

The random effects $\theta_{p}, \xi_{0 p}$ and $\log \left(\xi_{1 p}\right)$ are assumed to follow a multivariate normal distribution:

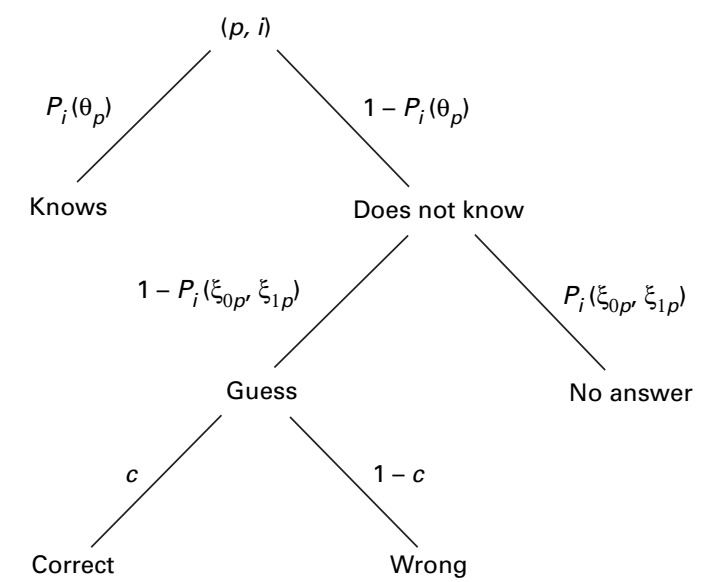

Fig. 2. Decision tree representation of the model 


$$
\left(\begin{array}{c}
\theta_{p} \\
\xi_{0 p} \\
\log \left(\xi_{1 p}\right)
\end{array}\right) \sim N_{3}(\boldsymbol{\mu}, \Omega)
$$

with $\boldsymbol{\mu}^{\prime}=\left(0, \mu_{\xi_{0}}, \mu_{\xi_{1}}\right)$ and $\Omega$ a positive definite covariance matrix. Given that $\xi_{1 p}>0$, we assumed that normality holds for $\log \left(\xi_{1 p}\right)$. This implies that $\xi_{1 p}$ is log-normally distributed. Conditional on the random effects $\theta_{p}, \xi_{0 p}$ and $\xi_{1 p}$, the responses of examinee $p$ to the $I$ items are assumed independent.

Under the model proposed, the probability of an omission (4) increases with the difficulty of the item, the initial propensity to omit answers and the sensitivity to test speededness, but it decreases with the examinee's ability.

Some remarks apply. First, the probability of a missing value depends on unobserved information (the random effects) and hence missingness is allowed to be MNAR. Second, the dropout and measurement processes are allowed to have some parameters in common, turning the model into a so-called shared parameter model. This implies that, apart from the correct or wrong answers, missingness also contains information about the difficulty of the item and personal ability. Third, if $P_{i}\left(\xi_{0 p}, \xi_{1 p}\right)=0$, the model proposed reduces to a three-parameter logistic in the case that $P_{i}\left(\theta_{p}\right)$ is given by equation (2) and to a one-parameter logistic model extended with guessing if $P_{i}\left(\theta_{p}\right)$ is given by equation (1). Fourth, if $P_{i}\left(\xi_{0 p}, \xi_{1 p}\right)>0, \pi_{p i 2}$ is smaller than the probability of a correct answer under either of these two logistic models. This follows immediately from a simple rearrangement of terms. Under the model proposed the probability of a correct answer is given by

$$
\pi_{p i 2}=P_{i}\left(\theta_{p}\right)+\left\{1-P_{i}\left(\xi_{0 p}, \xi_{1 p}\right)\right\} c\left\{1-P_{i}\left(\theta_{p}\right)\right\},
$$

whereas under the three-parameter model the success probability is given by

$$
P_{i}\left(\theta_{p}\right)+c\left\{1-P_{i}\left(\theta_{p}\right)\right\} .
$$

As a direct consequence, the lower asymptote (for $\theta_{p} \rightarrow-\infty$ ) of the model proposed, which is given by $\left\{1-P_{i}\left(\xi_{0 p}, \xi_{1 p}\right)\right\} c$, is smaller than the lower asymptote of the three-parameter or extended one-parameter logistic models (which is $c$ ).

We now report the results that were obtained on the SIMCE mathematics test data. Two models were fitted to the data: the model for test speededness as described above and a model without test speededness effects, i.e. a model with

$$
P_{i}\left(\xi_{0 p}, \xi_{1 p}\right)=P_{i}\left(\xi_{0 p}\right)=\frac{\exp \left(\xi_{0 p}\right)}{1+\exp \left(\xi_{0 p}\right)} .
$$

For both models $P_{i}\left(\theta_{p}\right)$ is given by the one-parameter logistic model and the analyses were performed under the assumption of independent random effects. The model parameters were estimated with the method of marginal maximum likelihood, as implemented in SAS procedure NLMIXED (Littell et al., 2006). The estimation of the reduced and the test speededness models (i.e. the maximization of the log-likelihood function and the computation of the inverse of the observed information matrix) took 4.5 and $38 \mathrm{~h}$ of central processor unit time respectively. All computations were performed on an Intel Pentium M 2.17 GHz computer with 1 Gbyte of random-access memory. The NLMIXED specifications were non-adaptive Gauss-Hermite quadrature with five quadrature points per dimension combined with a Newton-Raphson optimization algorithm. Example SAS code is given in Appendix A. In Table 1 the reduced model and the test speededness model are compared on the basis of $-2 l$, the Akaike information criterion AIC and the Bayesian information criterion BIC. Note that the reduced model is nested in the test speededness model and hence will always have a larger $-2 l$-value. The difference in 
Table 1. Goodness-of-fit statistics for the reduced and the test speededness model

\begin{tabular}{|c|c|c|c|c|c|c|c|c|c|c|}
\hline & \multicolumn{2}{|c|}{ All data } & \multicolumn{2}{|c|}{30 cases removed } & \multicolumn{2}{|c|}{60 cases removed } & \multicolumn{2}{|c|}{90 cases removed } & \multicolumn{2}{|c|}{120 cases removed } \\
\hline & Reduced & Speeded & Reduced & Speeded & Reduced & Speeded & Reduced & Speeded & Reduced & Speeded \\
\hline$-2 l$ & 184526 & 183996 & 181424 & 181156 & 178380 & 178212 & 175482 & 175361 & 172618 & 172591 \\
\hline AIC & 184630 & 184104 & 181528 & 181264 & 178484 & 178320 & 175586 & 175469 & 172722 & 172699 \\
\hline $\mathrm{BIC}$ & 184943 & 184428 & 181840 & 181588 & 178795 & 178644 & 175897 & 175792 & 173032 & 173021 \\
\hline
\end{tabular}

the $-2 l$-values can be used to construct a likelihood ratio test for the null hypothesis of the reduced model. Given a difference of 530, there is overwhelming evidence in favour of the test speededness model. Also the AIC and BIC indicate that the test speededness model is the most appropriate to describe the SIMCE mathematics test data.

To obtain an indication about the fit of the test speededness model to the SIMCE mathematics data, we also show in Fig. 1 the estimated theoretical proportions of omissions, wrong answers and correct answers (dotted curves), given by

$$
\begin{gathered}
P\left(Y_{p i 0}=1, Y_{p i 1}=0\right)=\int_{\mathbb{R}^{2}} \int_{0}^{\infty}\left\{1-P_{i}\left(\theta_{p}\right)\right\} P_{i}\left(\xi_{0 p}, \xi_{1 p}\right) \mathrm{d} F_{3}\left(\xi_{1 p}\right) \mathrm{d} F_{2}\left(\xi_{0 p}\right) \mathrm{d} F_{1}\left(\theta_{p}\right), \\
P\left(Y_{p i 0}=0, Y_{p i 1}=1\right)=(1-c) \int_{\mathbb{R}^{2}} \int_{0}^{\infty}\left\{1-P_{i}\left(\theta_{p}\right)\right\}\left\{1-P_{i}\left(\xi_{0 p}, \xi_{1 p}\right)\right\} \mathrm{d} F_{3}\left(\xi_{1 p}\right) \mathrm{d} F_{2}\left(\xi_{0 p}\right) \mathrm{d} F_{1}\left(\theta_{p}\right), \\
P\left(Y_{p i 0}=0, Y_{p i 1}=0\right)=c \int_{\mathbb{R}} \int_{0}^{\infty}\left\{1-P_{i}\left(\xi_{0 p}, \xi_{1 p}\right)\right\} \mathrm{d} F_{3}\left(\xi_{1 p}\right) \mathrm{d} F_{2}\left(\xi_{0 p}\right) \\
+\int_{\mathbb{R}^{2}} \int_{0}^{\infty}\left[1-\left\{1-P_{i}\left(\xi_{0 p}, \xi_{1 p}\right)\right\} c\right] P_{i}\left(\theta_{p}\right) \mathrm{d} F_{3}\left(\xi_{1 p}\right) \mathrm{d} F_{2}\left(\xi_{0 p}\right) \mathrm{d} F_{1}\left(\theta_{p}\right),
\end{gathered}
$$

with $F_{1}, F_{2}$ and $F_{3}$ denoting the distribution functions of examinee ability, initial propensity to omit answers and the examinee-specific effect of test speededness respectively, and with the unknown parameters replaced by their corresponding maximum likelihood estimates, as a function of the item number. As is clear from Fig. 1, the empirical and the estimated theoretical proportions agree quite well, indicating a good fit of the test speededness model. Note, however, that this goodness-of-fit evaluation involves only marginal probabilities and hence provides us with only a partial picture of the overall model fit. To evaluate further the overall goodness of fit we have used a parametric bootstrap approach. For this, we compare the empirical item characteristic curves (ICCs) with those which are obtained from repeated sampling from the proposed model for test speededness and omitted answers, and with parameters replaced by their maximum likelihood estimates. An ICC is a graph showing the probability of successfully answering an item as a function of the ability parameter. Here, we take a nonparametric approach with the abilities replaced by sum scores (i.e. we count for each examinee the number of correct answers and use this as a proxy for his or her ability) and with the probabilities of correctly answering the item replaced by the respective empirical proportions. If the model really fits the data, the observed ICCs should be in line with the simulated ICCs. The bootstrap procedure was implemented with a uniform $(-4,4)$ distribution for the person ability parameters. This choice was made also to obtain reliable estimates of the ICCs in the lower and upper ranges of ability. In Fig. 3 we show for some items the empirical ICCs (full curves) together with those obtained from 
100 bootstrap iterations (circles). As is clear from this plot, all empirical ICCs are contained in the $95 \%$ confidence band based on the bootstrap samples (broken curves), providing further evidence in favour of the model fit. For items 5 and 25, the empirical ICCs are different from the bootstrapped ICCs and indicate the need to include discrimination parameters. Although possible, we did not adjust the model in this direction:

(a) as in every simultaneous hypothesis testing situation some false positive results can be expected,

(b) given the size of the data set, two misfitting items out of 48 will probably not have a large effect on the inferences and

(c) although a model with discrimination parameters for these misfitted items may be useful to gain insight, in general, a modeller would not want to build such a contrived model.

The bootstrap goodness-of-fit results for the other items are similar to those which are shown in Fig. 3. To assess the effect of these two items, we refitted both models to the data set excluding them and noted that there was virtually no effect on the results.

In Fig. 4, the item difficulty estimates that were obtained under the reduced model (full curve) are compared with those from fitting the test speededness model (broken curve). Clearly, the two sets of item difficulty estimates are almost indistinguishable. Table 2 contains the estimates of the parameters of the random-effect distributions and the random guessing parameter $c$ under the reduced model. The corresponding estimates for the test speededness model are shown in Table 3. In Table $3, \mu_{\xi_{1}}$ and $\sigma_{\xi_{1}}^{2}$ are the mean and the variance respectively of $\log \left(\xi_{1 p}\right)$. The mean and the variance of $\xi_{1 p}$ are related to $\mu_{\xi_{1}}$ and $\sigma_{\xi_{1}}^{2}$ in the following way:

$$
\begin{gathered}
E\left(\xi_{1 p}\right)=\exp \left(\mu_{\xi_{1}}+\sigma_{\xi_{1}}^{2} / 2\right), \\
\operatorname{var}\left(\xi_{1 p}\right)=\exp \left\{2\left(\mu_{\xi_{1}}+\sigma_{\xi_{1}}^{2}\right)\right\}-\exp \left(2 \mu_{\xi_{1}}+\sigma_{\xi_{1}}^{2}\right),
\end{gathered}
$$

leading to the estimates $\widehat{E\left(\xi_{1 p}\right)}=0.4193(0.0663)$ and $\widehat{\operatorname{var}\left(\xi_{1 p}\right)}=0.8809(0.0580)$. The standard errors of these estimates were obtained by the delta method.

To obtain an idea about the possible gains that are achieved by explicitly modelling the nonresponse mechanism, we also fitted an extended one-parameter logistic model to a data set of complete cases. In Fig. 4 we show, next to the item difficulty estimates that were obtained under

Table 2. Parameter estimates under the extended one-parameter logistic model (complete-case analysis)

\begin{tabular}{|c|c|c|c|c|c|c|c|c|}
\hline \multirow[t]{3}{*}{ Parameter } & \multirow{2}{*}{\multicolumn{2}{|c|}{$\begin{array}{l}\text { Results from the } \\
\text { extended one-parameter } \\
\text { logistic model }\end{array}$}} & \multicolumn{6}{|c|}{ Results from the reduced model } \\
\hline & & & \multicolumn{2}{|c|}{ All data } & \multicolumn{2}{|c|}{60 cases removed } & \multicolumn{2}{|c|}{120 cases removed } \\
\hline & Estimate & Standard error & Estimate & $\begin{array}{l}\text { Standard } \\
\text { error }\end{array}$ & Estimate & $\begin{array}{l}\text { Standard } \\
\text { error }\end{array}$ & Estimate & $\begin{array}{c}\text { Standard } \\
\text { error }\end{array}$ \\
\hline$\sigma_{\theta}^{2}$ & 1.0327 & 0.0373 & 0.9928 & 0.0324 & 0.9924 & 0.0326 & 0.9894 & 0.0327 \\
\hline$\mu_{\xi_{0}}$ & - & - & -5.3783 & 0.0750 & -5.5185 & 0.0742 & -6.0216 & 0.1559 \\
\hline$\sigma_{\varepsilon_{\varepsilon}}^{2}$ & - & - & 3.4854 & 0.1083 & 2.7337 & 0.1064 & 5.9796 & 0.5413 \\
\hline$c^{\xi_{0}}$ & 0.1484 & 0.0055 & 0.1472 & 0.0050 & 0.1457 & 0.0051 & 0.1451 & 0.0051 \\
\hline
\end{tabular}
and the reduced model (all data, 60 or 120 locally most influential cases removed) 

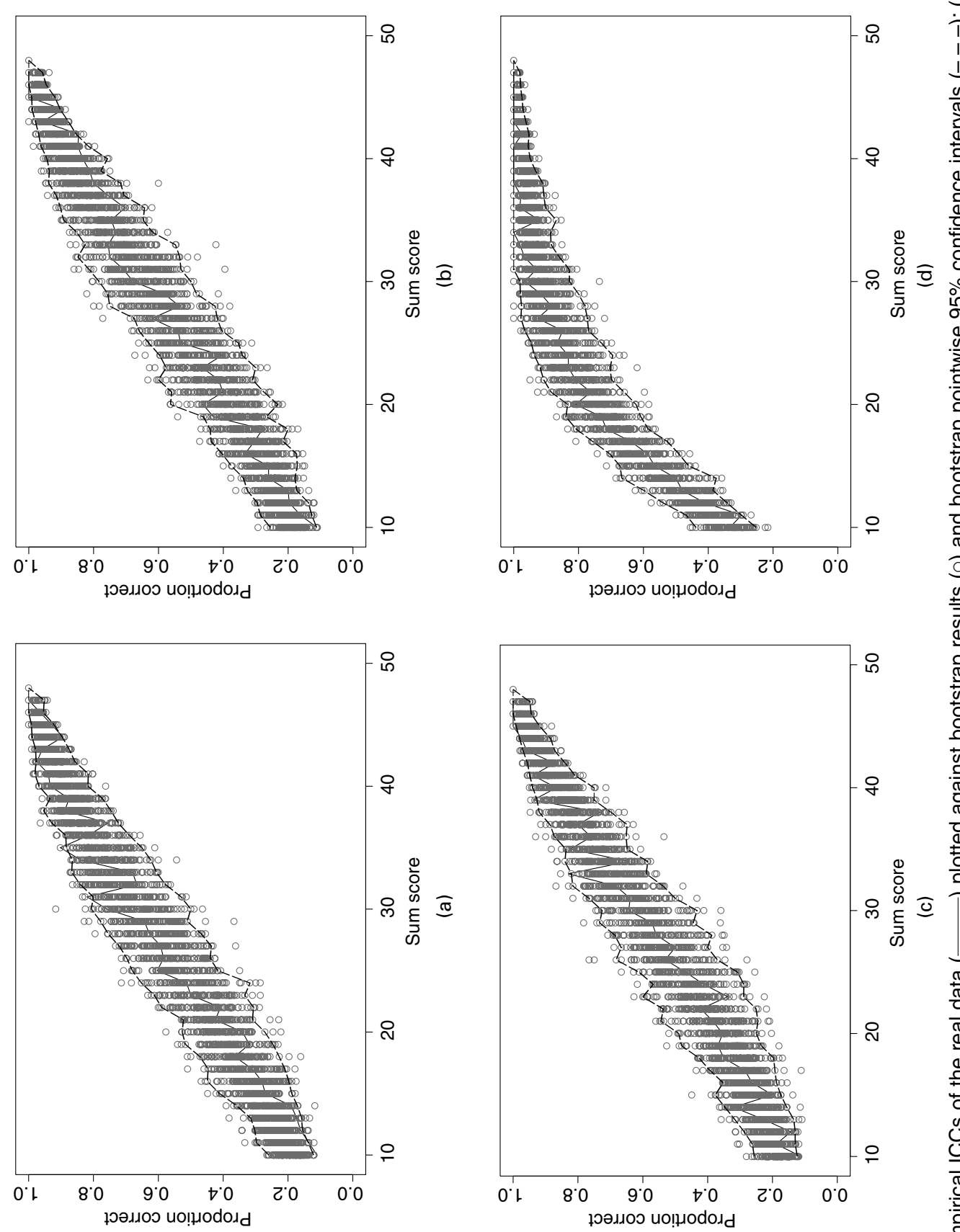

요

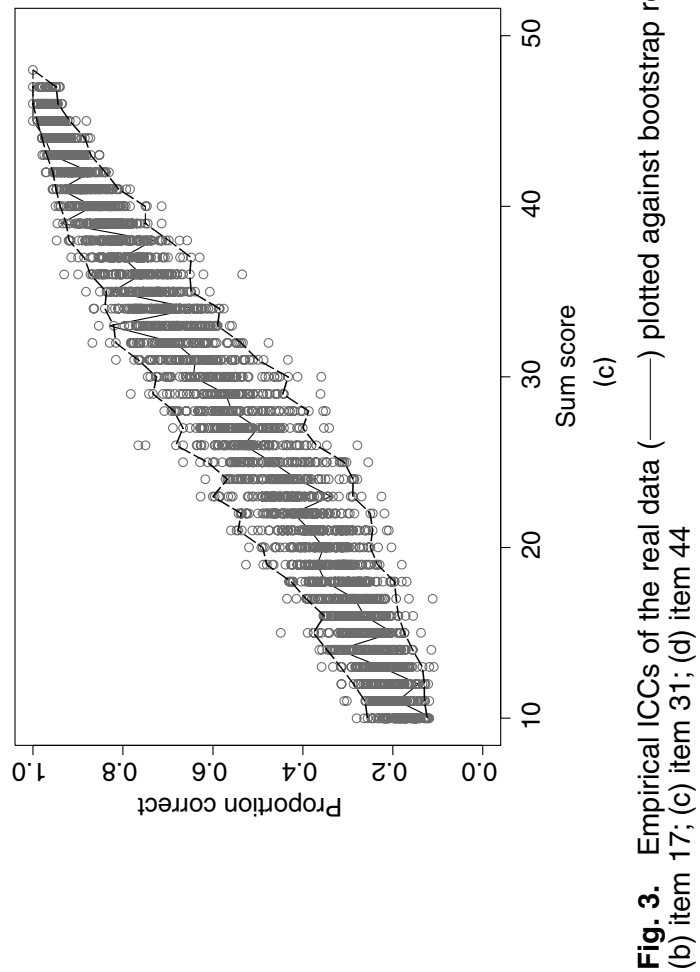




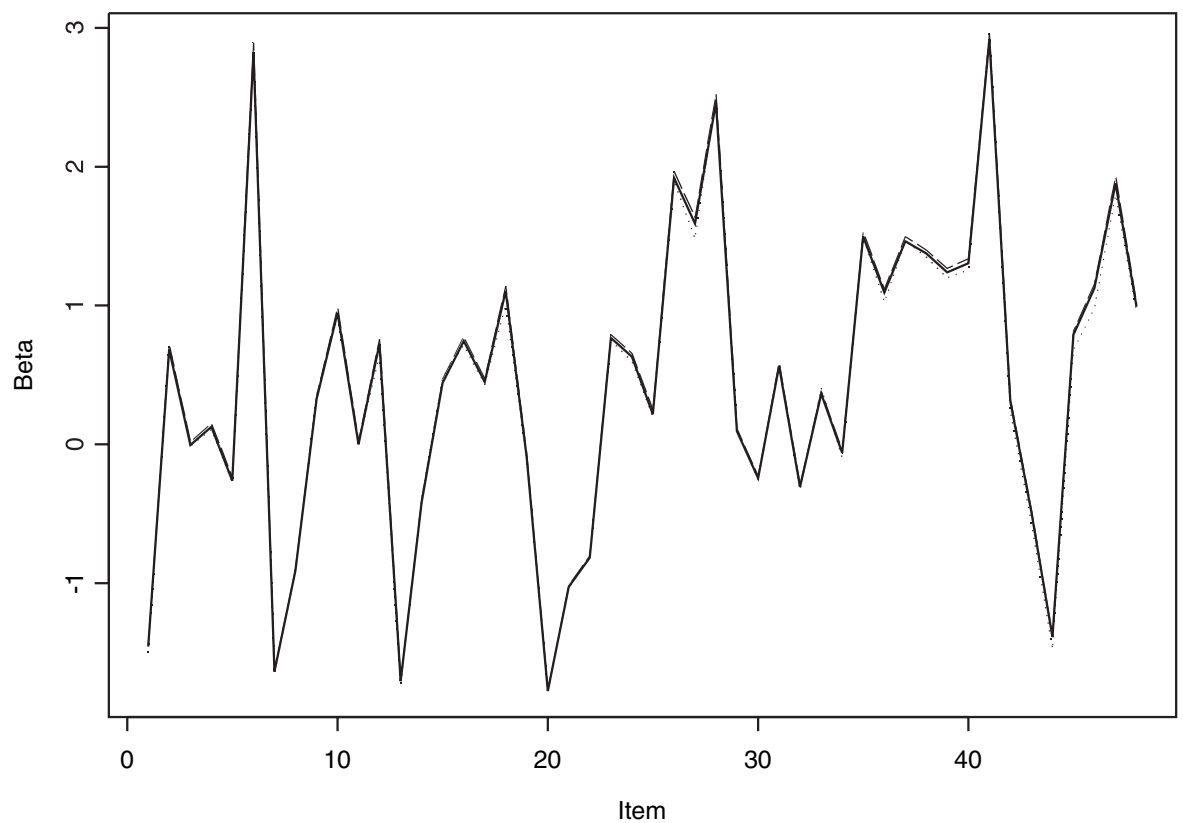

Fig. 4. Estimated difficulties of items under the reduced model (-), the model with test speededness $(--)$ and the extended one-parameter logistic model $(\cdots \cdots)$

Table 3. Parameter estimates under the test speededness model

\begin{tabular}{|c|c|c|c|c|c|c|}
\hline \multirow[t]{2}{*}{ Parameter } & \multicolumn{2}{|c|}{ All data } & \multicolumn{2}{|c|}{60 cases removed } & \multicolumn{2}{|c|}{120 cases removed } \\
\hline & Estimate & Standard error & Estimate & Standard error & Estimate & Standard error \\
\hline$\sigma_{\theta}^{2}$ & 1.0155 & 0.0330 & 0.9947 & 0.0327 & 0.9897 & 0.0327 \\
\hline$\mu_{\xi_{0}}$ & -5.7481 & 0.0965 & -5.5463 & 0.0751 & -6.1343 & 0.1740 \\
\hline$\sigma_{\xi_{0}}^{2}$ & 3.4794 & 0.1372 & 2.6475 & 0.1109 & 6.0364 & 0.6206 \\
\hline$\mu_{\xi_{1}}$ & -1.7657 & 0.2845 & -17.0993 & 9.6461 & -16.5640 & 15.3140 \\
\hline$\sigma_{\xi_{1}}^{2}$ & 1.7933 & 0.2558 & 44.7525 & 45.1727 & 37.2875 & 65.4619 \\
\hline$c^{\zeta 1}$ & 0.1524 & 0.0049 & 0.1463 & 0.0051 & 0.1453 & 0.0051 \\
\hline
\end{tabular}

the reduced model and the test speededness model, also those obtained from this additional model (dotted curve). Although small, the difference (the estimate under the test speededness model versus the estimate under the extended one-parameter logistic model) is in general positive and tends to increase with item number. This positive difference becomes more pronounced after normalization (Fig. 5(a)). The difference between the point estimates is normalized by the square root of the sum of the variances of the respective estimators, which is quite a conservative estimate of the true standard error and hence the true normalized differences will be larger. For instance, assuming a correlation between the two sets of $\boldsymbol{\beta}$-estimates of only 0.5 yields already two very significant and three borderline significant differences, whereas for a correlation of 0.75 these numbers are 8 and 2 respectively. The positive difference in point estimates can be explained quite easily. Under a complete-case analysis, incomplete response profiles are removed 


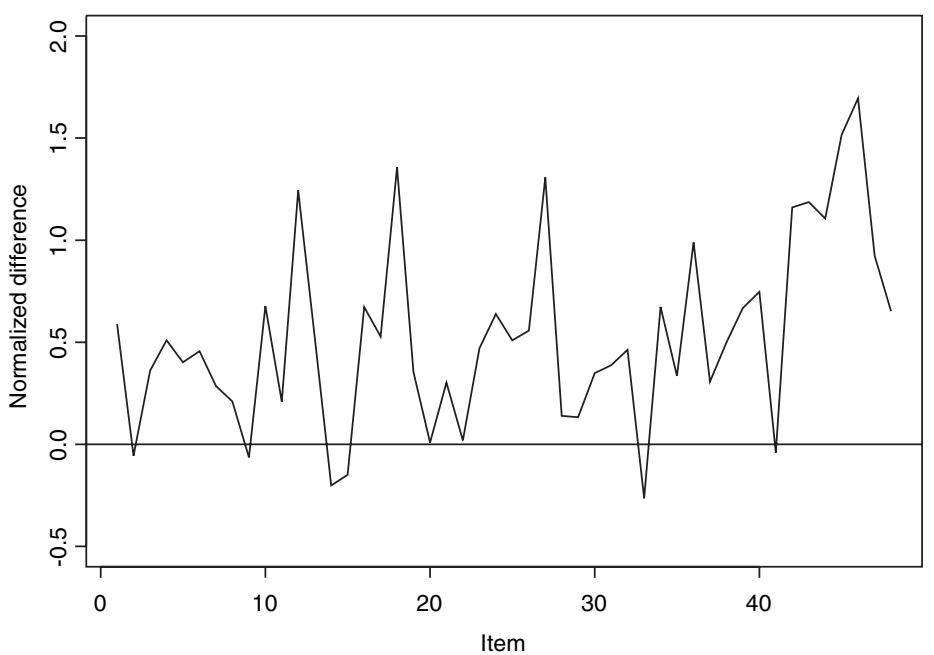

(a)

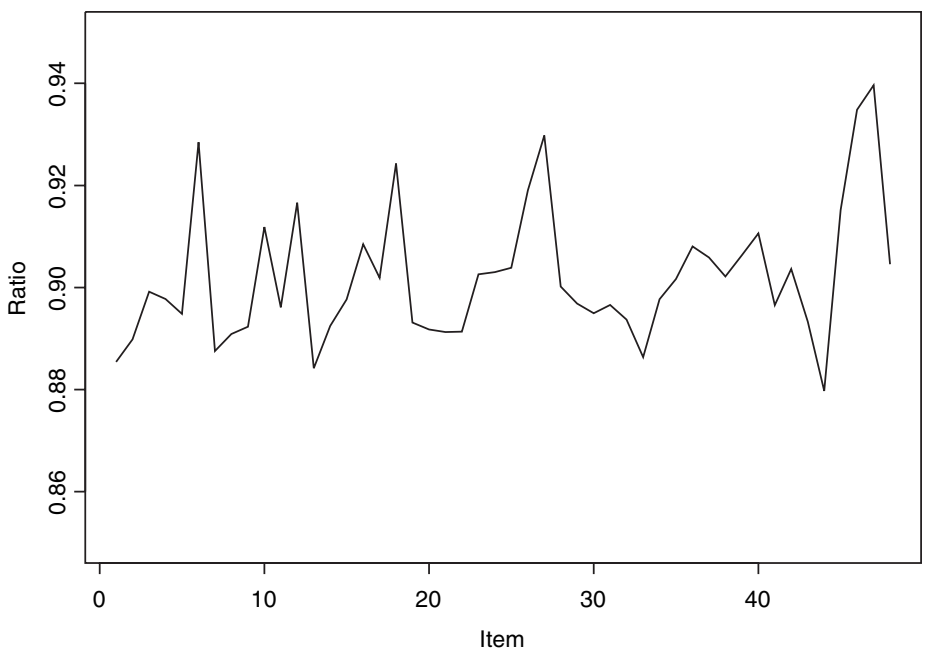

(b)

Fig. 5. (a) Normalized difference in estimates of the difficulty of items and (b) relative efficiency of the estimates for the item difficulty parameters: test speededness model versus extended one-parameter logistic model

and hence cannot contribute to the estimation of the difficulty of the items. In contrast, under the model proposed the non-response depends on the difficulty of the items (see equation (4)), and hence, besides correct and wrong answers, non-response also will contribute to the estimation of the difficulty of the items. Since under the model proposed the relationship between the item difficulty and the proportion of omissions is positive, the item difficulty estimate will tend to be larger than the estimate that is obtained under the extended one-parameter logistic model.

Similarly, the column for the extended one-parameter logistic model in Table 2 contains the point estimates and standard errors for $\sigma_{\theta}^{2}$ and $c$. As is clear from Table 2 and Table 3, the point estimates that are obtained under this model are very similar to the results that are obtained under the reduced model and the test speededness model. 
Apart from the effect on the point estimates, we also consider the effect of explicitly modelling non-response on the standard errors. Recall that for the SIMCE mathematics test data approximately $20 \%$ of the profiles contain at least one non-response and hence a complete-case analysis entails a substantial loss of information. This is illustrated in Fig. 5(b), where we show the ratio of the standard errors of the estimates of item difficulty under the test speededness and extended one-parameter logistic model. Explicitly modelling the non-response mechanism yields a gain in efficiency of about $10 \%$.

\section{Sensitivity analysis}

Sensitivity analysis as referred to at the end of Section 1 can take various forms. We are concerned with a sensitivity analysis allowing for the identification of subjects who have an inordinate effect on inferences about the key model parameters. Apart from global influence analysis, local influence has gained in popularity, thanks to the seminal work of Cook (1986) and various applications that are based thereon.

\subsection{Global influence diagnostics}

Global influence diagnostics are based on a case deletion approach (Chatterjee and Hadi, 1988). Broadly, all or part of a subject's measurements are deleted and key aspects of the model refitted, such as the likelihood value and parameter estimates. When the distance between the overall and the refitted measure is large in a precisely defined sense, a case is considered to be influential. Global influence or case deletion diagnostics have been well developed, for example, for linear regression and explicit forms derived. Two of the main problems with the method that is applied to more general settings are that

(a) the application of the method can be computer intensive since no closed form expressions exist and

(b) it may be difficult to gain further insight into why a certain subject, observation or set of observations is influential.

\subsection{Local influence diagnostics}

We shall introduce the local influence method in generic terms. For this, assume that we have a model, parameterized by a vector-valued parameter $\boldsymbol{\theta}$. Given a set of observed data, let $l(\boldsymbol{\theta})$ denote the log-likelihood function of the model postulated, where $\boldsymbol{\theta}$ is a $p$-dimensional vector of model parameters. The model postulated is perturbed by introducing a $q$-dimensional vector $\boldsymbol{\omega}$ which is assumed to belong to some open subset $\tilde{\Omega}$ of $\mathbb{R}^{q}$ and the log-likelihood function of the perturbed model is denoted by $l(\theta \mid \omega)$. We further assume that there is an $\boldsymbol{\omega}_{0} \in \tilde{\Omega}$ such that $l(\boldsymbol{\theta})=l\left(\boldsymbol{\theta} \mid \boldsymbol{\omega}_{0}\right)$ for all $\boldsymbol{\theta}$. Let $\hat{\boldsymbol{\theta}}$ be the maximum likelihood estimator for $\boldsymbol{\theta}$, which is obtained by maximizing $l(\boldsymbol{\theta})$, and let $\hat{\boldsymbol{\theta}}_{\boldsymbol{\omega}}$ denote the maximum likelihood estimator for $\boldsymbol{\theta}$ under $l(\boldsymbol{\theta} \mid \boldsymbol{\omega})$. Finally, assume that $l(\boldsymbol{\theta} \mid \boldsymbol{\omega})$ is twice continuously differentiable in $(\boldsymbol{\theta}, \boldsymbol{\omega})$.

The local influence approach compares $\hat{\boldsymbol{\theta}}$ and $\hat{\boldsymbol{\theta}}_{\omega}$. Similar estimates indicate that the parameter estimates are stable with respect to the proposed perturbations of the model postulated. Strongly different estimates indicate that the estimation procedure is highly sensitive with respect to perturbations. Cook (1986) proposed to measure the distance between $\hat{\boldsymbol{\theta}}$ and $\hat{\boldsymbol{\theta}}_{\boldsymbol{\omega}}$ by the likelihood displacement, which is defined by

$$
\operatorname{LD}(\boldsymbol{\omega})=2\left\{l(\hat{\boldsymbol{\theta}})-l\left(\hat{\boldsymbol{\theta}}_{\boldsymbol{\omega}}\right)\right\} .
$$


Note that the log-likelihood function of the model postulated is evaluated in both $\hat{\boldsymbol{\theta}}$ and $\hat{\boldsymbol{\theta}}_{\boldsymbol{\omega}}$ and hence $\operatorname{LD}(\omega) \geqslant 0$. Note also that the likelihood displacement takes the variability of $\hat{\boldsymbol{\theta}}$ into account. Indeed, $\operatorname{LD}(\boldsymbol{\omega})$ will be large if $l(\boldsymbol{\theta})$ is strongly curved at $\hat{\boldsymbol{\theta}}$, which means that $\boldsymbol{\theta}$ is estimated with high precision. From this perspective, a graph of $\operatorname{LD}(\boldsymbol{\omega})$ versus $\boldsymbol{\omega}$ contains essential information on the influence of the perturbation scheme of interest. It is useful to view this graph as the geometric surface that is formed by the $(q+1)$-dimensional vector

$$
\alpha(\boldsymbol{\omega})=\left(\begin{array}{c}
\boldsymbol{\omega} \\
\operatorname{LD}(\boldsymbol{\omega})
\end{array}\right)
$$

as $\omega$ varies throughout $\tilde{\Omega}$. Since this surface, the so-called influence graph, can only be depicted when $q \leqslant 2$, Cook (1986) proposed to look at normal curvatures of $\alpha(\boldsymbol{\omega})$ in $\boldsymbol{\omega}_{0}$ in a direction $\mathbf{l}$, with $\mathbf{I}$ a $q$-dimensional vector of unit length. These normal curvatures can easily be calculated as

$$
C_{\mathbf{l}}=2\left|\mathbf{l}^{\prime} \Delta^{\prime} \ddot{L}^{-1} \Delta \mathbf{l}\right|
$$

with

$$
\ddot{L}=\left.\frac{\partial^{2} l(\boldsymbol{\theta})}{\partial \boldsymbol{\theta} \partial \boldsymbol{\theta}^{\prime}}\right|_{\boldsymbol{\theta}=\hat{\boldsymbol{\theta}}}
$$

and $\Delta$ a $p \times q$ matrix of which the $i$ th column $\Delta_{i}$ is given by

$$
\Delta_{i}=\left.\frac{\partial^{2} l(\boldsymbol{\theta} \mid \boldsymbol{\omega})}{\partial \boldsymbol{\theta} \partial \omega_{i}}\right|_{\boldsymbol{\theta}=\hat{\boldsymbol{\theta}}, \omega=\omega_{0}} .
$$

The normal curvature (8) can be used in several ways to study the influence graph $\alpha(\omega)$, each corresponding to a particular direction $\mathbf{l}$ in $\tilde{\Omega}$. One evident choice is the vector $\mathbf{l}_{i}$ which has a 1 on position $i$ and 0 s elsewhere, corresponding to a perturbation of the postulated model by weight $\omega_{i}$ only. In this case equation (8) reduces to

$$
C_{i}=2\left|\Delta_{i}^{\prime} \ddot{L}^{-1} \Delta_{i}\right|
$$

Other important directions are the directions of minimal and maximal curvature, denoted $\mathbf{l}_{\min }$ and $\mathbf{l}_{\max }$ respectively, which are obtained as solutions to the minimization and maximization respectively of $C_{\mathbf{l}}$ over the space of all vectors of unit length. It can be shown that $C_{\mathbf{l}_{\min }}$ and $C_{\mathbf{l}_{\max }}$ correspond to the smallest and largest eigenvalues of $-2 \Delta^{\prime} \ddot{L}^{-1} \Delta$ and $\mathbf{l}_{\min }$ and $\mathbf{l}_{\max }$ are the corresponding eigenvectors.

So far, the discussion of local influence diagnostics has focused on the complete $\boldsymbol{\theta}$-vector. Similar ideas can be applied to obtain the local influence of perturbations on subsets of $\boldsymbol{\theta}$. Suppose that we partition $\boldsymbol{\theta}=\left(\boldsymbol{\theta}_{1}^{\prime}, \boldsymbol{\theta}_{2}^{\prime}\right)^{\prime}$, where $\boldsymbol{\theta}_{i}$ is a vector of dimension $p_{i}$, and agree that interest is in only $\boldsymbol{\theta}_{1}$. For this case, Cook (1986) showed that the analogue of equation (7) is given by

$$
\mathrm{LD}_{s}(\boldsymbol{\omega})=2\left[l(\hat{\boldsymbol{\theta}})-l\left\{\hat{\boldsymbol{\theta}}_{1 \boldsymbol{\omega}}, g\left(\hat{\boldsymbol{\theta}}_{1 \boldsymbol{\omega}}\right)\right\}\right],
$$

where $g$ is the function maximizing $l\left(\boldsymbol{\theta}_{1}, \boldsymbol{\theta}_{2}\right)$ for each fixed $\boldsymbol{\theta}_{1}, \hat{\boldsymbol{\theta}}_{1 \boldsymbol{\omega}}$ is determined from the partition $\hat{\boldsymbol{\theta}}_{\boldsymbol{\omega}}=\left(\hat{\boldsymbol{\theta}}_{1 \omega}^{\prime}, \hat{\boldsymbol{\theta}}_{2 \omega}^{\prime}\right)^{\prime}$ and the influence graph is the geometric surface that is formed by the vector 


$$
\alpha_{s}(\omega)=\left(\begin{array}{c}
\omega \\
\operatorname{LD}_{s}(\boldsymbol{\omega})
\end{array}\right) .
$$

Completely analogously to the case of the complete vector $\theta$, the study of the local influence of perturbations on $\hat{\boldsymbol{\theta}}_{1}$ is based on the normal curvatures of $\alpha_{s}(\boldsymbol{\omega})$. These are now given by

$$
C_{\mathbf{l}}\left(\boldsymbol{\theta}_{1}\right)=2\left|\mathbf{I}^{\prime} \Delta^{\prime}\left(\ddot{L}^{-1}-B_{22}\right) \Delta \mathbf{I}\right|,
$$

with

$$
B_{22}=\left(\begin{array}{cc}
0 & 0 \\
0 & L_{22}^{-1}
\end{array}\right)
$$

and $L_{22}$ determined from the partition

$$
\ddot{L}=\left(\begin{array}{ll}
L_{11} & L_{12} \\
L_{21} & L_{22}
\end{array}\right)
$$

where

$$
L_{i j}=\left.\frac{\partial^{2} l(\boldsymbol{\theta})}{\partial \boldsymbol{\theta}_{i} \partial \boldsymbol{\theta}_{j}^{\prime}}\right|_{\boldsymbol{\theta}=\hat{\boldsymbol{\theta}}}, \quad i, j=1,2 .
$$

\subsection{Sensitivity analysis of the item response theory model with omitted responses}

In this section, we elaborate on a possible perturbation scheme of the proposed model for omitted responses. In particular, as interest is in the effects of test speededness, we examine which individuals have the largest effect on the parameter estimates when we introduce test speededness in their response profile. To do so, we extend the model by including perturbation parameters $\omega_{p}, p=1, \ldots, P$, in the probability of an omitted item in the following way:

$$
P_{i}\left(\xi_{0 p}, \xi_{1 p} \mid \omega_{p}\right)=\frac{\exp \left(\xi_{0 p}+\omega_{p} \xi_{1 p} i / I\right)}{1+\exp \left(\xi_{0 p}+\omega_{p} \xi_{1 p} i / I\right)} .
$$

We then examine how the likelihood changes when making an infinitesimal change away from $\boldsymbol{\omega}:=\left(\omega_{1}, \ldots, \omega_{P}\right)=\mathbf{0}$ in a particular direction $\mathbf{l}$. We consider the case where $P_{i}\left(\theta_{p}\right)$ is given by a one-parameter logistic model.

Denote by $\boldsymbol{\theta}$ the parameter vector of the model postulated (the model at $\boldsymbol{\omega}=\mathbf{0}$ ), so $\boldsymbol{\theta}^{\prime}=$ $\left(\beta_{1}, \ldots, \beta_{I}, \sigma_{\theta}^{2}, \mu_{\xi_{0}}, \sigma_{\xi_{0}}^{2}, \sigma_{12}, c\right)$ with $\sigma_{12}=\operatorname{cov}\left(\theta, \xi_{0}\right)$, and by $\mathbf{Y}_{p}$ the $I \times 2$ matrix of responses of examinee $p, \mathbf{Y}_{p}=\left(\left(Y_{p 10}, Y_{p 11}\right), \ldots,\left(Y_{p I 0}, Y_{p I 1}\right)\right)^{\prime}$. The perturbation scheme as defined above involves, besides $\boldsymbol{\theta}$, also the parameters $\mu_{\xi_{1}}, \sigma_{\xi_{1}}^{2}, \sigma_{13}$ and $\sigma_{23}$, where $\sigma_{13}=\operatorname{cov}\left(\theta, \xi_{1}\right)$ and $\sigma_{23}=$ $\operatorname{cov}\left(\xi_{0}, \xi_{1}\right)$. These additional parameters must be fixed by the user since, following the discussion in Section 3.2, only estimates for the components of $\boldsymbol{\theta}$ are obtained from a null model fit. However, this more general parameterization allows us to assess the effect of perturbing the postulated model with an extra random effect, in particular a random test speededness effect, that may be correlated with the random effects in the model postulated. If we are interested only in the effect of perturbing the model with a fixed, i.e. non-random, test speededness effect, we simply fix $\sigma_{13}$ and $\sigma_{23}$ at 0 . Doing so, the mean of $\xi_{1 p}$ appears as a common scale factor in the expressions for the elements of $\Delta$ and hence can be safely ignored. This follows immediately from the expressions in Appendix B. 
The log-likelihood function of the perturbed model is given by

$$
\begin{aligned}
l(\boldsymbol{\theta} \mid \boldsymbol{\omega}) & =\sum_{p=1}^{P} l_{p}\left(\boldsymbol{\theta} \mid \omega_{p}\right) \\
& =\sum_{p=1}^{P} \ln \left\{P\left(\mathbf{Y}_{p}=\mathbf{y}_{p} \mid \omega_{p}\right)\right\},
\end{aligned}
$$

with

$$
P\left(\mathbf{Y}_{p}=\mathbf{y}_{p} \mid \omega_{p}\right)=\int_{\mathbb{R}^{2}} \int_{0}^{\infty} A_{p}\left(\omega_{p}\right) f\left(\theta_{p}, \xi_{0 p}, \xi_{1 p}\right) \mathrm{d} \xi_{1 p} \mathrm{~d} \xi_{0 p} \mathrm{~d} \theta_{p},
$$

and

$$
\begin{aligned}
A_{p}\left(\omega_{p}\right) & =P\left(\mathbf{Y}_{p}=\mathbf{y}_{p} \mid \theta_{p}, \xi_{0 p}, \xi_{1 p}, \omega_{p}\right) \\
& =\prod_{i=1}^{I} P\left(\mathbf{Y}_{p i}=\mathbf{y}_{p i} \mid \theta_{p}, \xi_{0 p}, \xi_{1 p}, \omega_{p}\right) \\
& =\prod_{i=1}^{I} \pi_{p i 0}\left(\omega_{p}\right)^{y_{p i 0}} \pi_{p i 1}\left(\omega_{p}\right)^{y_{p i 1}} \pi_{p i 2}\left(\omega_{p}\right)^{1-y_{p i 0}-y_{p i 1}} .
\end{aligned}
$$

Hence, $\pi_{p i 0}\left(\omega_{p}\right), \pi_{p i 1}\left(\omega_{p}\right)$ and $\pi_{p i 2}\left(\omega_{p}\right)$ are given by equations (4), (5) and (6) respectively, with $P_{i}\left(\xi_{0 p}, \xi_{1 p}\right)$ replaced by $P_{i}\left(\xi_{0 p}, \xi_{1 p} \mid \omega_{p}\right)$.

The calculation of the local influence measures can be carried out as soon as expressions for $\ddot{L}$ and $\Delta$ have been obtained. The elements of $\ddot{L}$ are not computed analytically as these can easily be obtained from the maximization of $l(\theta)$, for instance by using the SAS NLMIXED procedure. Indeed, using the SAS output delivery system functionality we can easily obtain the Hessian evaluated at the maximum likelihood estimate, as computed by NLMIXED, and export it to a data file. For details we refer to Appendix A. The elements of the columns $\Delta_{p}$ of $\Delta$ are given in Appendix B. In the case of independent random effects, if the response profile of examinee $p$ contains only omitted and wrong answers, the elements of $\Delta_{p}$ related to the parameters of classical IRT models, i.e. $\boldsymbol{\beta}, \sigma_{\theta}^{2}$ and $c$, are 0 , as shown in the following proposition. This means that the respective mixed derivatives of such examinees do not contribute to the local influence for the parameters in question.

Proposition 1. Let $\mathbf{Y}_{p}=\left(\left(Y_{p 10}, Y_{p 11}\right), \ldots,\left(Y_{p I 0}, Y_{p I 1}\right)\right)$ denote the response profile of examinee $p$. Assume that, conditional on the random effects $\theta_{p}, \xi_{0 p}$ and $\xi_{1 p}$, the bivariate random vectors $\left(Y_{p i 0}, Y_{p i 1}\right), i=1, \ldots, I$, are independent, with conditional probability functions given by

$$
\begin{gathered}
P\left(Y_{p i 0}=1, Y_{p i 1}=0 \mid \theta_{p}, \xi_{0 p}, \xi_{1 p}, \omega_{p}\right)=\left\{1-P_{i}\left(\theta_{p}\right)\right\} P_{i}\left(\xi_{0 p}, \xi_{1 p} \mid \omega_{p}\right), \\
P\left(Y_{p i 0}=0, Y_{p i 1}=1 \mid \theta_{p}, \xi_{0 p}, \xi_{1 p}, \omega_{p}\right)=\left\{1-P_{i}\left(\theta_{p}\right)\right\}\left\{1-P_{i}\left(\xi_{0 p}, \xi_{1 p} \mid \omega_{p}\right)\right\}(1-c), \\
P\left(Y_{p i 0}=0, Y_{p i 1}=0 \mid \theta_{p}, \xi_{0 p}, \xi_{1 p}, \omega_{p}\right)= \\
\left\{1-P_{i}\left(\xi_{0 p}, \xi_{1 p} \mid \omega_{p}\right)\right\} c \\
+\left[1-\left\{1-P_{i}\left(\xi_{0 p}, \xi_{1 p} \mid \omega_{p}\right)\right\} c\right] P_{i}\left(\theta_{p}\right),
\end{gathered}
$$

where

$$
\begin{aligned}
P_{i}\left(\theta_{p}\right) & =\frac{\exp \left(\theta_{p}-\beta_{i}\right)}{1+\exp \left(\theta_{p}-\beta_{i}\right)}, \\
P_{i}\left(\xi_{0 p}, \xi_{1 p} \mid \omega_{p}\right) & =\frac{\exp \left(\xi_{0 p}+\omega_{p} \xi_{1 p} i / I\right)}{1+\exp \left(\xi_{0 p}+\omega_{p} \xi_{1 p} i / I\right)} .
\end{aligned}
$$




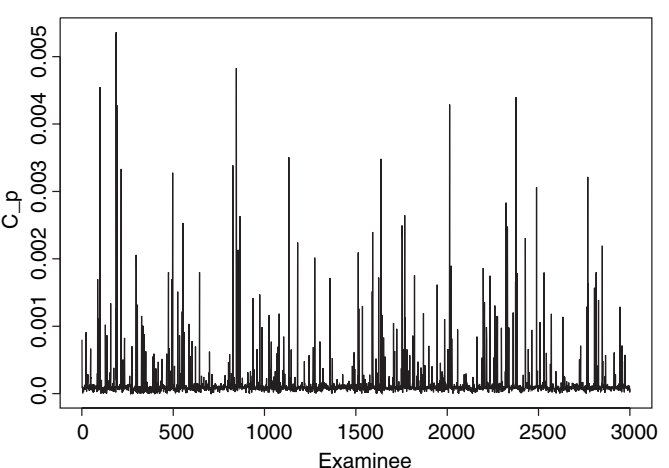

(a)

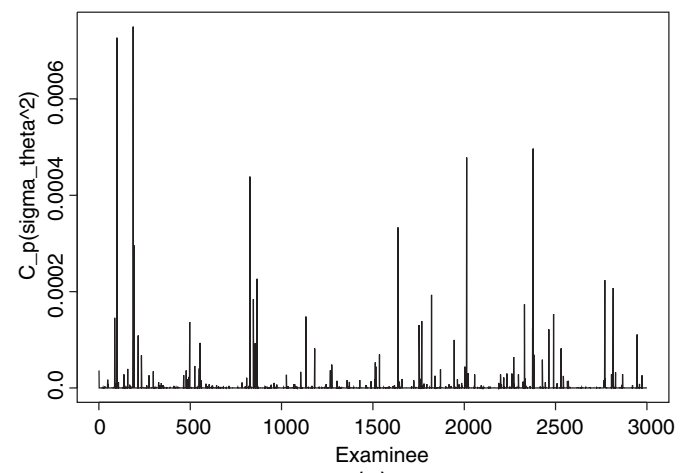

(c)

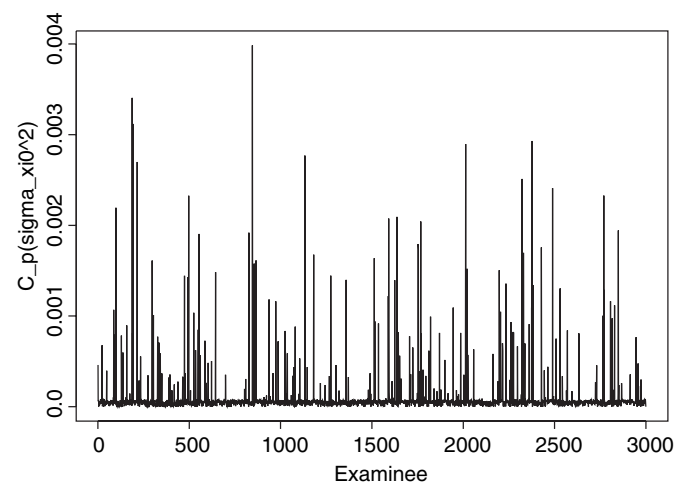

(e)

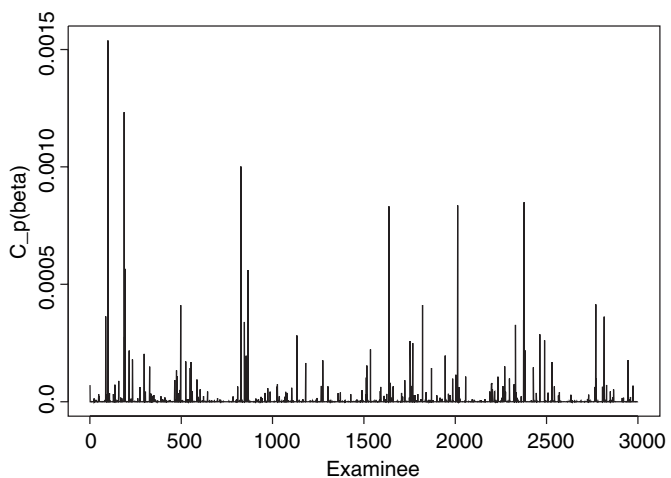

(b)

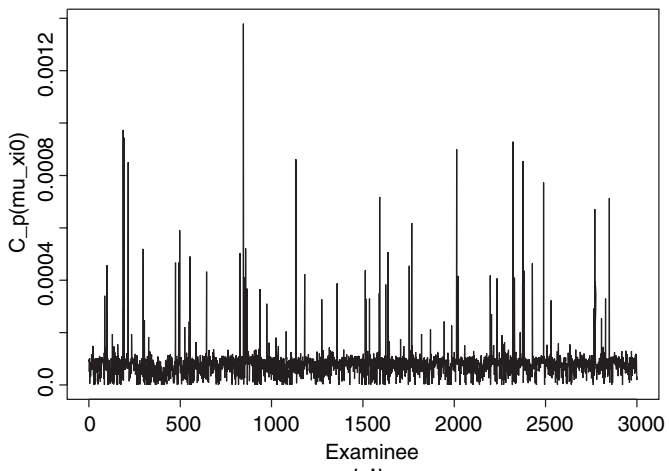

(d)

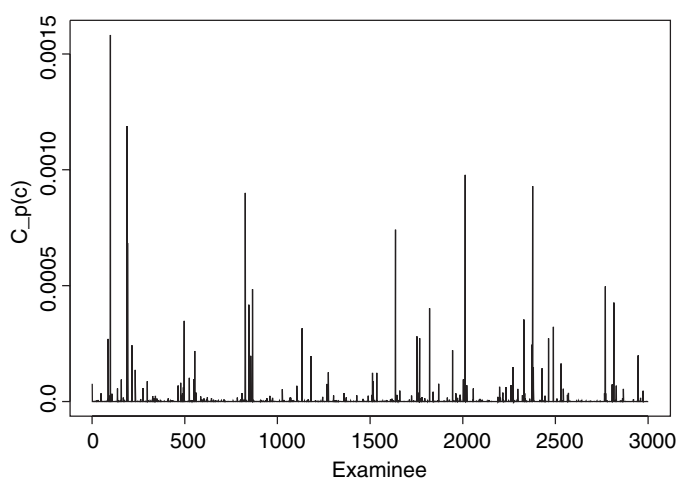

(f)

Fig. 6. Index plots of (a) $C_{p}$, (b) $C_{p}(\boldsymbol{\beta})$, (c) $C_{p}\left(\sigma_{\theta}^{2}\right)$, (d) $C_{p}\left(\mu_{\xi_{0}}\right)$, (e) $C_{p}\left(\sigma_{\xi_{0}}^{2}\right)$ and (f) $C_{p}(c)$

Further, assume that

$$
\left(\begin{array}{c}
\theta_{p} \\
\xi_{0 p} \\
\log \left(\xi_{1 p}\right)
\end{array}\right) \sim N_{3}(\boldsymbol{\mu}, \Omega),
$$

with $\boldsymbol{\mu}^{\prime}=\left(0, \mu_{\xi_{0}}, \mu_{\xi_{1}}\right)$ and $\Omega=\operatorname{diag}\left(\sigma_{\theta}^{2}, \sigma_{\xi_{0}}^{2}, \sigma_{\xi_{1}}^{2}\right)$. Then, denoting by $l_{p}$ the log-likelihood contribution of examinee $p$, if the response profile of examinee $p$ contains only omitted and wrong answers, we have 

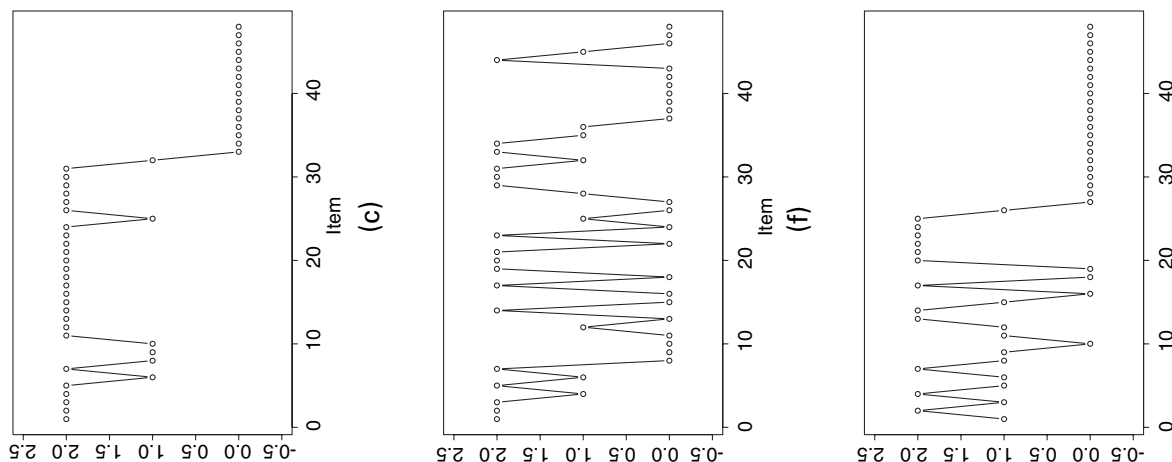

त्रें

ठํㅇ

00

II II

$\mathrm{C}^{2} \mathrm{e}^{2}$

ริ ซิ

.

焉

एक

ब苟

E्ञ

$\underline{\underline{\Phi}} \equiv$

षै.

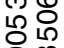

0

11 .

ô 11
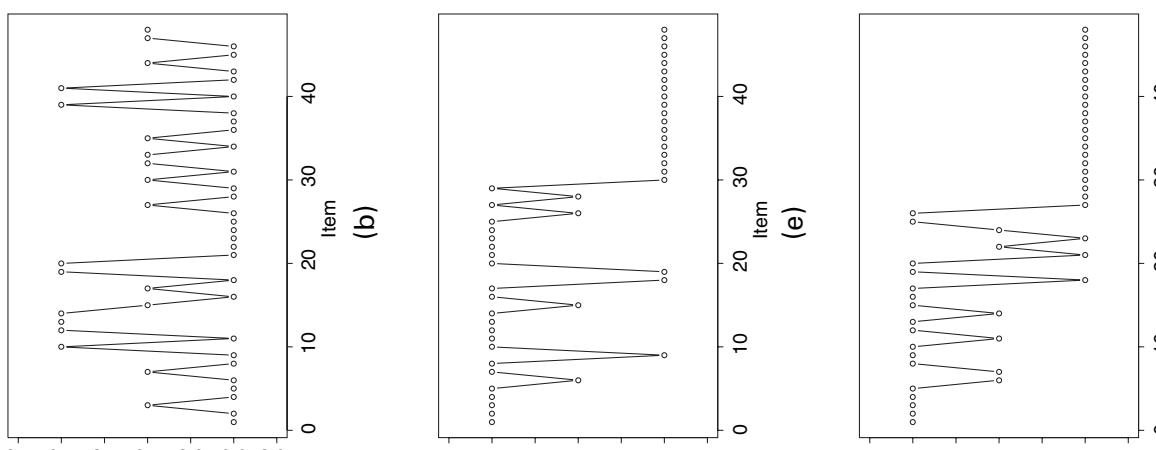

$\infty 0$

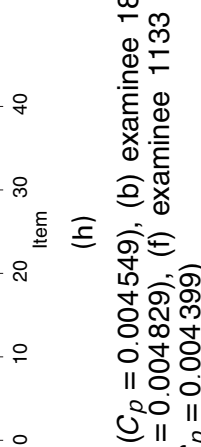

110

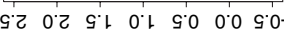
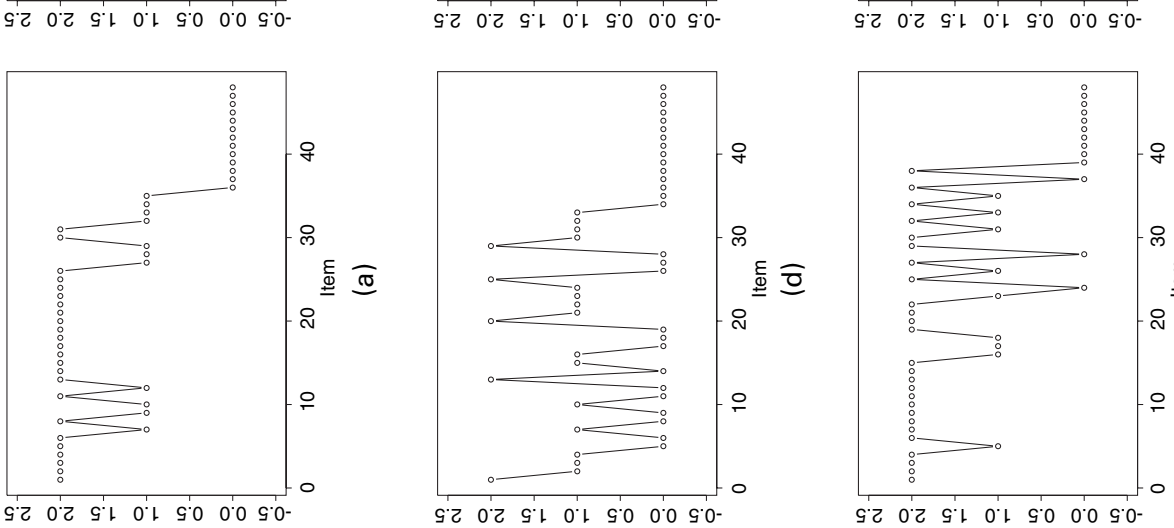

80

$\Phi 0 \hat{m}$

\&

๙० 0

$\times$

ब实

E

흥

흐용

뜽

둥

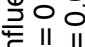

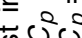

"艹o

हल

응

ㅈN

잉 $₫$

है है

ベ㐅

운으로 

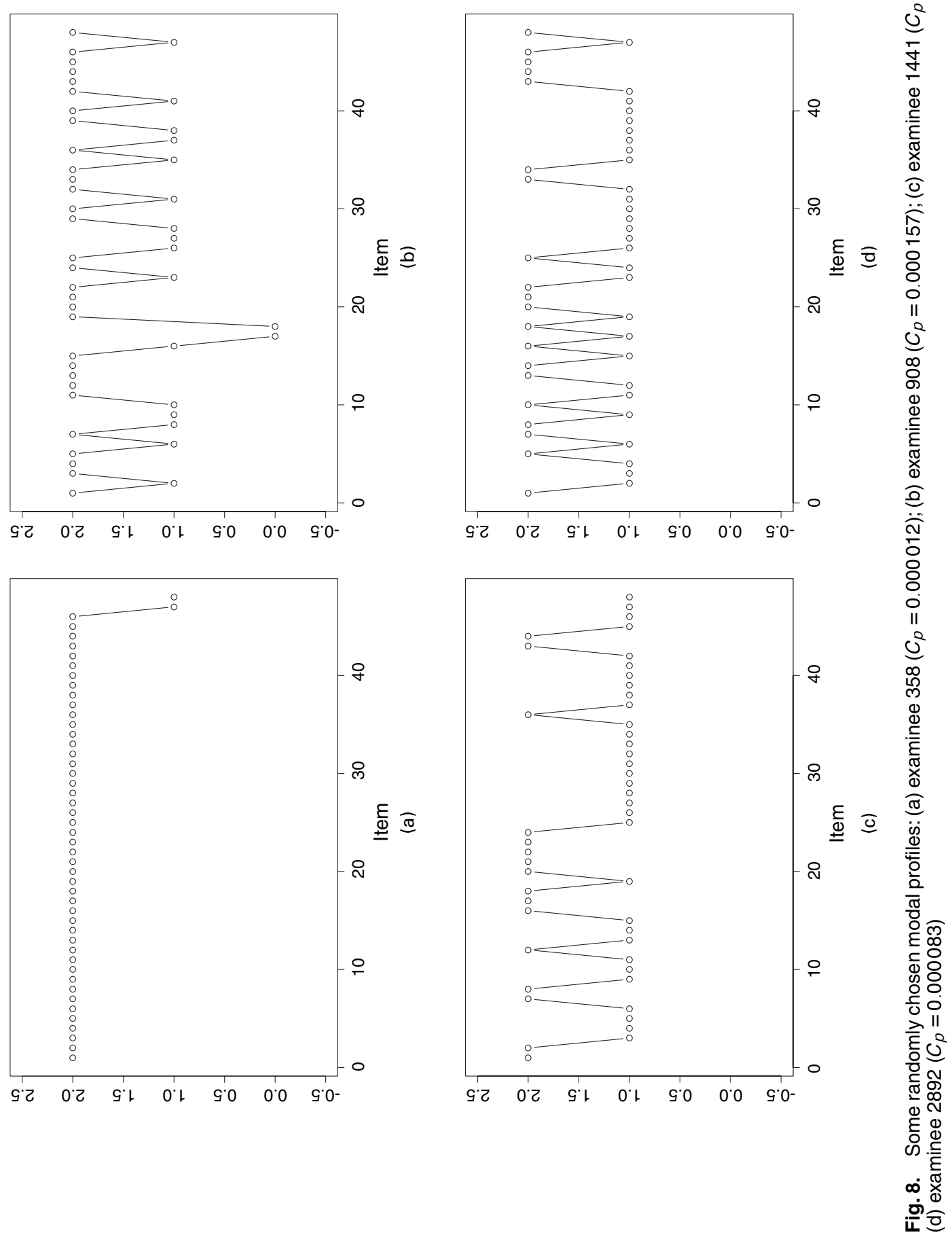
Table 4. Correlation between the normal curvatures and the proportion of omissions $(p)$, the proportion of omissions in the first half of the test $\left(p_{1}\right)$, the proportion of omissions in the second half of the test $\left(p_{2}\right)$ and the variance of the responses $(\operatorname{var}(Y)) \dagger$

\begin{tabular}{|lccccc|}
\hline Measure & $p$ & $p_{1}$ & $p_{2}$ & $\operatorname{var}(Y)$ & $R^{2}$ \\
\hline$C_{p}$ & 0.8941 & 0.6677 & 0.8990 & 0.8068 & 0.8300 \\
$C_{p}(\boldsymbol{\beta})$ & 0.6248 & 0.4070 & 0.6631 & 0.5390 & 0.4400 \\
$C_{p}\left(\sigma_{\theta}^{2}\right)$ & 0.5545 & 0.3342 & 0.6043 & 0.4529 & 0.3676 \\
$C_{p}\left(\mu_{\xi_{0}}\right)$ & 0.7052 & 0.4929 & 0.7289 & 0.6445 & 0.5420 \\
$C_{p}\left(\sigma_{\xi_{0}}^{2}\right)$ & 0.8827 & 0.6524 & 0.8915 & 0.7764 & 0.8063 \\
$C_{p}(c)$ & 0.5552 & 0.3156 & 0.6162 & 0.4714 & 0.3808 \\
\hline
\end{tabular}

$\dagger$ The column $R^{2}$ gives the coefficient of determination from regressing the curvatures on $p_{2}$ and $\operatorname{var}(Y)$.

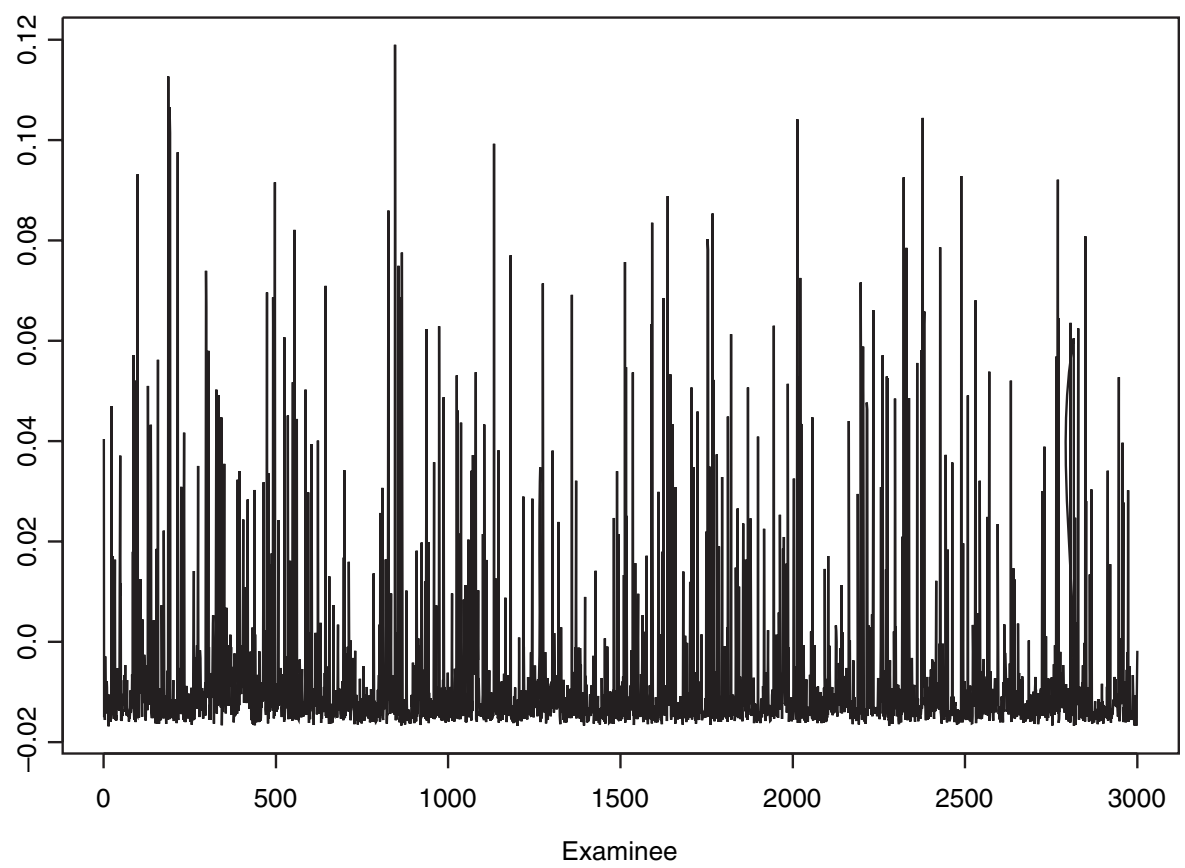

Fig. 9. Components of the direction of maximal curvature, I $\max$

$$
\begin{gathered}
\left.\frac{\partial^{2} l_{p}\left(\boldsymbol{\theta} \mid \omega_{p}\right)}{\partial \beta_{i} \partial \omega_{p}}\right|_{\omega_{p}=0}=0, \quad i=1, \ldots, I, \\
\left.\frac{\partial^{2} l_{p}\left(\boldsymbol{\theta} \mid \omega_{p}\right)}{\partial \sigma_{\theta}^{2} \partial \omega_{p}}\right|_{\omega_{p}=0}=0, \\
\left.\frac{\partial^{2} l_{p}\left(\boldsymbol{\theta} \mid \omega_{p}\right)}{\partial c \partial \omega_{p}}\right|_{\omega_{p}=0}=0 .
\end{gathered}
$$


Proof. The elements of $\Delta_{p}$ in the case of independent random effects are given in Appendix B. Evaluating these expressions at a response profile that contains only missing values and wrong answers yields the result of the proposition.

The authors developed a Fortran program to compute the elements of $\Delta$, the normal curvatures $C_{\mathbf{l}}$ and the direction of maximal curvature $\mathbf{l}_{\max }$. In this program the numerical integrations are performed by Numerical Algorithms Group library subroutines D01BBF and D01FBF, and the direction of maximal curvature is computed by using subroutine F02FCF (Numerical Algorithms Group, 1993). Besides the local influence for the complete-parameter vector $\boldsymbol{\theta}$, we also consider the item difficulty parameters, the variance of personal ability, the mean and variance of the propensity to omit answers and the random-guessing parameter.

\subsection{Sistema di Medición de la Calidad de la Educación mathematics test data}

Finally, we discuss the results of local influence in the reduced model, applied to the SIMCE mathematics test data set. As stated in Section 3.3, the intention of the analysis is to identify the examinees for whom introducing test speededness will have a large effect on the parameter estimates or, otherwise stated, to identify the examinees who are responsible for the test speededness conclusion. This can be achieved in several ways. The first possibility is to consider the normal curvatures of $\operatorname{LD}(\boldsymbol{\omega})$ in $\boldsymbol{\omega}=\mathbf{0}$ in the direction $\mathbf{l}_{p}$, denoted $C_{p}$, where $\mathbf{l}_{p}$ is a $P$-dimensional vector with a 1 in position $p$ and 0 s elsewhere, corresponding to a perturbation of the postulated model by $\omega_{p}$ alone, i.e. introducing test speededness for examinee $p$ alone. Fig. 6(a) shows the index plot of the $C_{p}$ diagnostic measure. Examinees with a large $C_{p}$ are clearly important since for these examinees introducing test speededness will have a large effect on the parameter estimates. The response profiles of the nine locally most influential examinees are shown in Fig. 7, with omitted items, incorrect answers and correct answers coded as 0,1 and 2 respectively. For ease of comparison, we also show four randomly chosen modal response profiles in Fig. 8. Compared with the non-influential profiles, all highlighted response profiles contain many omitted answers, especially for end-of-test items. This of course directly reflects the way that test speededness is incorporated into the 'extended' model: $\xi_{1 p}$ is assumed to be a positive random effect, and hence the probability of omission increases with the position of the item. The response profiles of the other locally influential examinees are comparable with those shown in Fig. 7, of course with decreasing evidence for test speededness with decreasing curvature $C_{p}$. The computation of the $C_{p}$ diagnostic measure for $p=1, \ldots, 3000$, using 64 quadrature points per dimension, took about $10 \mathrm{~h}$ of central processor unit time. To illustrate the strength of the relationship between the normal curvatures and the proportions of answers omitted, in particular omissions at the end of the test, we show in Table 4 the correlation between the normal curvatures and the proportions of answers omitted, and the proportions of answers omitted in the first and second half of the test respectively. Clearly, $C_{p}$ and $p_{2}$ have a very strong positive relationship. In addition, we also show the correlation with the variance of the response variable $Y$. The $C_{p}$-measure correlates quite highly with this characteristic also. A possible explanation is that, given the relatively high variability of the difficulties of the items, examinees with quite variable response profiles contain more information about $\boldsymbol{\theta}$, i.e. they have a log-likelihood contribution that is more strongly curved at $\hat{\boldsymbol{\theta}}$ than those with less variable profiles. A linear regression of the curvatures on the proportions of answers omitted in the second half of the test and the sample variances of the responses indicates that $83 \%$ of the total variation of the curvatures can be explained by variation in the latter two factors.

The effect of introducing test speededness on subsets of $\boldsymbol{\theta}$ can be assessed in a completely analogous fashion. In Figs 6(b), 6(c), 6(d), 6(e) and 6(f) we show the index plots of $C_{p}(\boldsymbol{\beta})$, 
$C_{p}\left(\sigma_{\theta}^{2}\right), C_{p}\left(\mu_{\xi_{0}}\right), C_{p}\left(\sigma_{\xi_{0}}^{2}\right)$ and $C_{p}(c)$ respectively. Clearly, for some subsets the index plots look very similar. On the one hand we have the index plots of parameters that are related to classical IRT models, in particular $\boldsymbol{\beta}, \sigma_{\theta}^{2}$ and $c$, whereas on the other hand we have those that are related to the parameters describing the non-response probability, here $\mu_{\xi_{0}}$ and $\sigma_{\xi_{0}}^{2}$. Note that the index plots of the latter two parameters are very similar to the index plot for $C_{p}$, which is confirmed by correlations of 0.84 and 0.98 respectively. Given these considerations it is not surprising that $C_{p}\left(\mu_{\xi_{0}}\right)$ and $C_{p}\left(\sigma_{\xi_{0}}^{2}\right)$ also correlate quite highly with the proportion of answers omitted in the second half of the test, at least considerably higher than $C_{p}(\boldsymbol{\beta}), C_{p}\left(\sigma_{\theta}^{2}\right)$ and $C_{p}(c)$; see Table 4. Despite this fact, the sets of locally influential examinees that are identified by the latter curvatures overlap to a large extent with the set that is identified by $C_{p}$.

A further approach to the identification of locally influential examinees is based on the direction of maximal curvature, $\mathbf{l}_{\max }$. This direction shows how to perturb the reduced model so as to obtain the largest local changes in likelihood displacement. Observe that this method allows us to identify groups of examinees that are jointly influential, perhaps to varying degrees. Fig. 9 displays the components of this direction, in the case that interest is in local influence assessment for the complete $\boldsymbol{\theta}$-vector, for the SIMCE mathematics test data. Clearly, in our case this plot conveys essentially the same information regarding local influence as the $C_{p}$ index plot (correlation 0.88).

Finally, we illustrate the effect that the locally influential observations have on the estimation results by removing the 30, 60, 90 and 120 locally most influential observations from the analysis. The removal took place on the basis of the direction of maximal curvature $\mathbf{l}_{\max }$ as this direction allows us to identify the subjects who jointly exert a large influence. Table 1 contains the goodness-of-fit statistics $-2 l$, AIC and BIC for both the reduced and the test speededness model after removal of influential observations. As is clear from the results, the differences between the goodness-of-fit statistics of both models become smaller as more locally influential observations are removed, with finally, i.e. after removal of 120 profiles, virtually no difference in fit on the basis of the criterion BIC. Tables 2 and 3 contain the estimates for the parameters of the random-effects distributions and the random-guessing parameter $c$ under the reduced and test speededness model respectively. These results clearly illustrate the large influence of a rather small subset of locally influential observations on the estimates of the parameters that are related to the non-response mechanism. In particular, we stress the instability of the estimates for $\mu_{\xi_{1}}$ and $\sigma_{\xi_{1}}^{2}$ after removing $2 \%$ and $4 \%$ respectively of the observations. Although the initial results clearly pointed in the direction of an overall speededness, the local influence analysis puts these results in a different perspective as they may be driven by rather a small subset of locally influential profiles.

\section{Discussion and conclusion}

In this paper, we developed an IRT model to explain non-response in test data. The model was derived from a decision tree describing the student's states and actions when she or he encounters an item. Application of the model postulated to the SIMCE mathematics test data taught us that

(a) non-response depends on an examinee-specific propensity to omit answers in the case that one does not know the answer,

(b) non-response tends to occur more often at end-of-test items, a fact that may be explained by test speededness, and

(c) the ultimate effect of being closer to the end of the test is subject specific. 
Compared with an analysis based on an extended one-parameter logistic model of complete profiles only, this approach, where missing observations are modelled simultaneously with the observed responses, yields improved parameter estimates, in the sense of reduced standard errors. However, caution is in order since the model proposed fits within the MNAR framework, and hence the conclusions are potentially strongly affected by the posited non-response mechanism. To assess the effect of the modelling assumptions on the parameter estimates we followed the local influence route. In particular we considered the effect of perturbing a reduced model with a random test speededness effect that may be correlated with the random effects that are present in the reduced model. From the results that were obtained on the SIMCE data set it became clear that the method successfully identifies the examinees who did not fit the reduced model because their response profiles were affected by test speededness effects. Moreover, we illustrated that the method also allowed us to assess the magnitude of the support for a particular model, in particular the test speededness model, by progressively removing locally influential profiles until estimation results become unstable.

Although local influence diagnostics have many advantages, some problems remain. For example, it would be useful to dispose of unambiguous cut-offs above which an observation or subject is considered influential. Lesaffre and Verbeke (1998) could show, in the linear mixed model context, that the sum of the influence values is approximately equal to $-2 N$, with $N$ the sample size. In other settings, such as that studied by Verbeke et al. (2001), such calibration was not possible. As a result, judgment is needed about the cut-off between influential and non-influential subjects. Arguably, a combination of the influence diagnostics and substantive knowledge, supported by plots of the candidate influential subjects, will be needed.

As pointed out by one of the referees, the current design does not allow for a definitive separation of the intrinsic difficulty of an item and the 'difficulty' that is due to test speededness. Specific designs, including randomization of the order in which the items are presented to the students, would be well in place. At the same time, the analysis of existing instruments with our proposed method, next to a conventional analysis, is of strong value as well.

The local influence diagnostics, which were advocated in this paper as tools for identifying examinees having a large influence on the parameter estimates when test speededness is introduced in their response profiles, may also offer interesting perspectives for person fit analysis. Person fit methods refer to statistical methods for evaluating the misfit of individual test performances to an item response model or other item-score patterns in a sample of people. In general, these methods do not allow recovery of the mechanism that created the deviant itemscore patterns (see for instance Meijer and Sijtsma (2001) for a good overview) and hence can be considered as the IRT analogues of the global influence diagnostics. However, some recent contributions explicitly test against specific violations of a test model assumption or particular types of deviant item-score patterns. An interesting example in this respect is the class of the so-called optimal person fit statistics that was proposed by Levine and Drasgow (1988). They used the Neyman-Pearson lemma to construct likelihood ratio tests for aberrant item-score patterns. Following the discussion in Section 3.2, the local influence analysis is based on normal curvatures of $\operatorname{LD}(\omega)$, which is a quantity that measures the distance between the maximum likelihood estimates obtained under the postulated model and the perturbed model in terms of the postulated model log-likelihood, and hence may be related to the optimal person fit statistic of Levine and Drasgow (1988). A formal study of this relationship is a topic of on-going research.

\section{Acknowledgements}

The research that is reported in this paper was supported by Interuniversity Attraction Poles 
P5/24, which was awarded to Paul De Boeck, Geert Molenberghs and Iven Van Mechelen, by GOA/2005/04, which was awarded to Paul De Boeck and Iven Van Mechelen, by BIL05/03, which was awarded to Paul De Boeck, Emmanuel Lesaffre and Geert Molenberghs, and by Fondo Nacional de Investigación Científica y Tecnológica 1060722 awarded to Guido del Pino, Ernesto San Martin and Fernando Quintana. The Sistema de Medición de la Calidad de la Educación Office from the Chilean Government kindly allowed us access to the databases that were used in this study.

\section{Appendix A: Example SAS code}

\section{A.1. Model without test speededness effect}

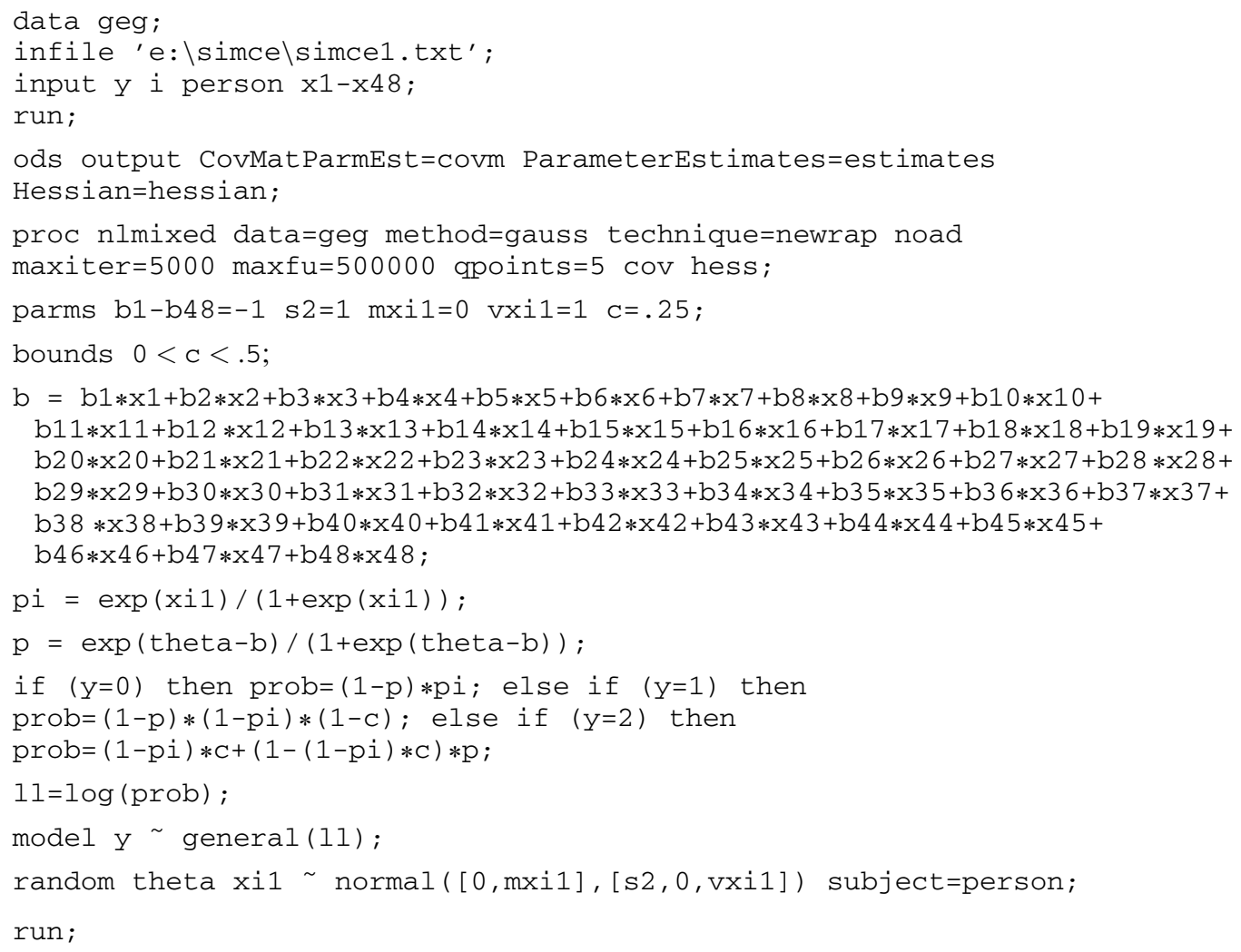

\section{A.2. Test speededness model}

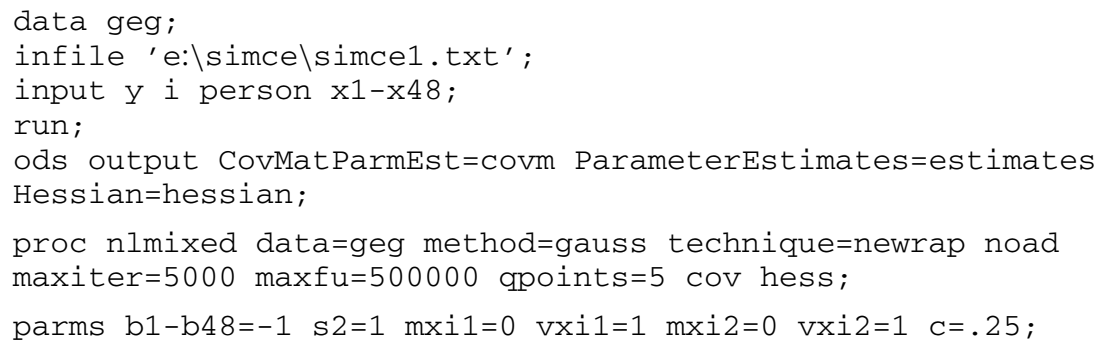




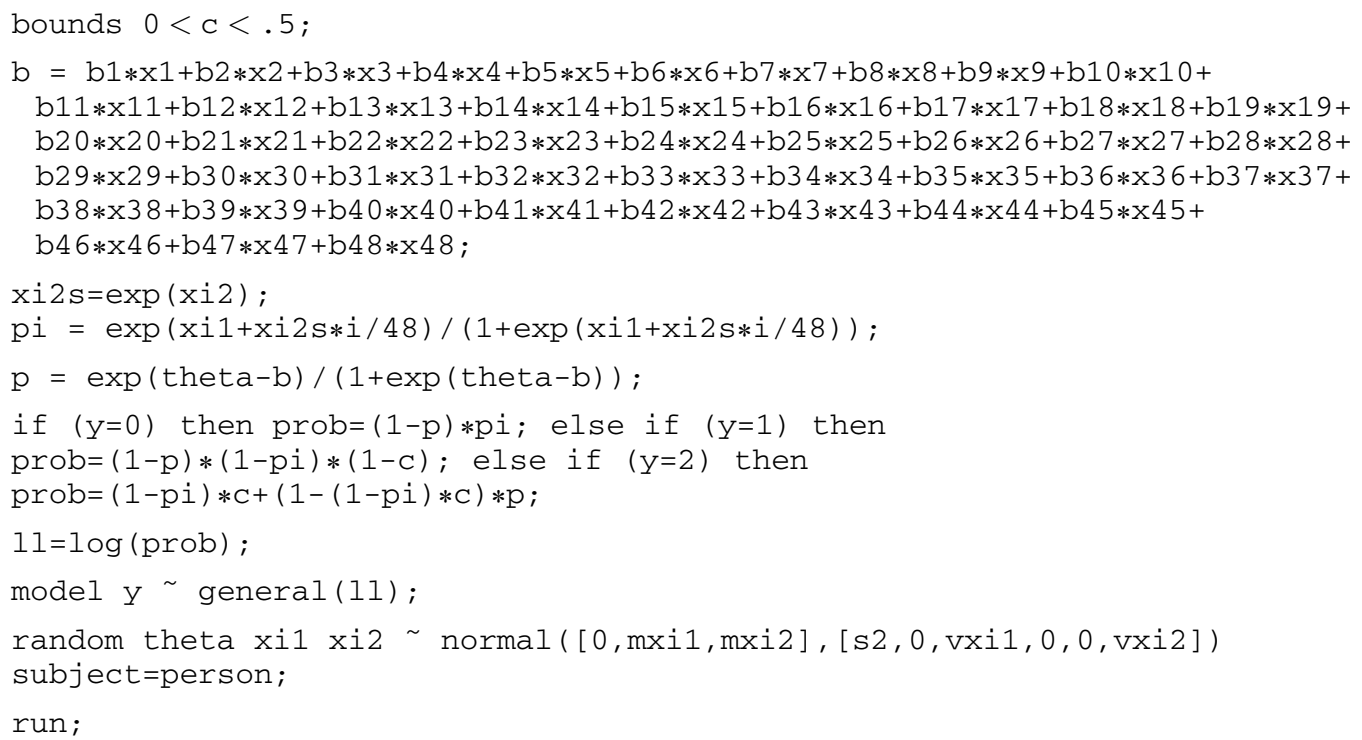

\section{Appendix B: Elements of $\Delta_{p}$}

The joint density function of the random effects $\theta_{p}, \xi_{0 p}$ and $\xi_{1 p}$ is given by

$$
f\left(\theta_{p}, \xi_{0 p}, \xi_{1 p}\right)=\frac{1}{(2 \pi)^{3 / 2}|\Omega|^{1 / 2} \xi_{1 p}} \exp \left(-\frac{\kappa^{\prime} \Omega^{-1} \kappa}{2}\right),
$$

with $\boldsymbol{\kappa}^{\prime}=\left(\theta_{p}, \xi_{0 p}-\mu_{\xi_{0}}, \ln \left(\xi_{1 p}\right)-\mu_{\xi_{1}}\right)$. Partition $\boldsymbol{\kappa}$ as $\boldsymbol{\kappa}=\left(\boldsymbol{\kappa}_{1}^{\prime}, \ln \left(\xi_{1 p}\right)-\mu_{\xi_{1}}\right)^{\prime}$, with $\boldsymbol{\kappa}_{1}^{\prime}=\left(\theta_{p}, \xi_{0 p}-\mu_{\xi_{0}}\right)$. Similarly, partition $\Omega$ as

with

$$
\Omega=\left(\begin{array}{ll}
\Omega_{11} & \Omega_{12} \\
\Omega_{12}^{\prime} & \Omega_{22}
\end{array}\right)
$$

$$
\begin{gathered}
\Omega_{11}=\left(\begin{array}{cc}
\sigma_{\theta}^{2} & \sigma_{12} \\
\sigma_{12} & \sigma_{\xi_{0}}^{2}
\end{array}\right), \\
\Omega_{12}=\left(\begin{array}{c}
\sigma_{13} \\
\sigma_{23}
\end{array}\right),
\end{gathered}
$$

and $\Omega_{22}=\sigma_{\xi_{1}}^{2}$. Integrating out $\xi_{1 p}$ from equation (10), we obtain the joint density function of $\theta_{p}$ and $\xi_{0 p}$ which is the density function of a bivariate normal distribution with mean vector $\left(0, \mu_{\xi_{0}}\right)$ and covariance matrix $\Omega_{11}$. Using the property of the inverse of a partitioned matrix and the basic properties of determinants, the conditional distribution of $\xi_{1 p}$ given $\theta_{p}$ and $\xi_{0 p}$ can be shown to be a log-normal distribution with parameters

$$
\mu=\mu_{\xi_{1}}+\Omega_{12}^{\prime} \Omega_{11}^{-1} \kappa_{1}
$$

and

$$
\sigma^{2}=\sigma_{\xi_{1}}^{2}-\Omega_{12}^{\prime} \Omega_{11}^{-1} \Omega_{12}
$$

The elements of $\Delta_{p}$ are given by

$$
\begin{gathered}
\left.\frac{\partial^{2} l_{p}\left(\boldsymbol{\theta} \mid \omega_{p}\right)}{\partial \beta_{i} \partial \omega_{p}}\right|_{\omega_{p}=0}=\frac{1}{I_{1 p}}\left\{-\frac{I_{2 p} I_{3 p i}}{I_{1 p}}+I_{4 p i}-\left(1-y_{p i 0}-y_{p i 1}\right) c \frac{i}{I} I_{5 p i}\right\}, \quad i=1, \ldots, I, \\
\left.\frac{\partial^{2} l_{p}\left(\boldsymbol{\theta} \mid \omega_{p}\right)}{\partial \sigma_{\theta}^{2} \partial \omega_{p}}\right|_{\omega_{p}=0}=\frac{1}{I_{1 p}}\left(-\frac{I_{2 p} I_{6 p}}{I_{1 p}}+I_{7 p}\right),
\end{gathered}
$$




$$
\begin{gathered}
\left.\frac{\partial^{2} l_{p}\left(\boldsymbol{\theta} \mid \omega_{p}\right)}{\partial \mu_{\xi_{0}} \partial \omega_{p}}\right|_{\omega_{p}=0}=\frac{1}{I_{1 p}}\left(-\frac{I_{2 p} I_{8 p}}{I_{1 p}}+I_{9 p}\right), \\
\left.\frac{\partial^{2} l_{p}\left(\boldsymbol{\theta} \mid \omega_{p}\right)}{\partial \sigma_{\xi_{0}}^{2} \partial \omega_{p}}\right|_{\omega_{p}=0}=\frac{1}{I_{1 p}}\left(-\frac{I_{2 p} I_{10 p}}{I_{1 p}}+I_{11 p}\right), \\
\left.\frac{\partial^{2} l_{p}\left(\boldsymbol{\theta} \mid \omega_{p}\right)}{\partial \sigma_{12} \partial \omega_{p}}\right|_{\omega_{p}=0}=\frac{1}{I_{1 p}}\left(-\frac{I_{2 p} I_{12 p}}{I_{1 p}}+I_{13 p}\right), \\
\left.\frac{\partial^{2} l_{p}\left(\boldsymbol{\theta} \mid \omega_{p}\right)}{\partial c \partial \omega_{p}}\right|_{\omega_{p}=0}=\frac{1}{I_{1 p}}\left(-\frac{I_{2 p} I_{14 p}}{I_{1 p}}+I_{15 p}-I_{16 p}\right),
\end{gathered}
$$

where

$$
I_{1 p}=\int_{\mathbb{R}^{2}} A_{p} f\left(\theta_{p}, \xi_{0 p}\right) \mathrm{d} \theta_{p} \mathrm{~d} \xi_{0 p}
$$

$$
\begin{aligned}
I_{2 p}= & \int_{\mathbb{R}^{2}} \frac{A_{p} \delta_{1}}{1+\exp \left(\xi_{0 p}\right)} \sum_{i=1}^{I} \frac{i}{I}\left[y_{p i 0}-y_{p i 1} \exp \left(\xi_{0 p}\right)-\frac{\left(1-y_{p i 0}-y_{p i 1}\right) c \exp \left(\xi_{0 p}\right)}{c\left\{1+\exp \left(\theta_{p}-\beta_{i}\right)\right\}+\left\{1-c+\exp \left(\xi_{0 p}\right)\right\} \exp \left(\theta_{p}-\beta_{i}\right)}\right] \\
& \times f\left(\theta_{p}, \xi_{0 p}\right) \mathrm{d} \theta_{p} \mathrm{~d} \xi_{0 p},
\end{aligned}
$$

$$
\begin{aligned}
& I_{3 p i}=\int_{\mathbb{R}^{2}} \frac{A_{p} \exp \left(\theta_{p}-\beta_{i}\right)}{1+\exp \left(\theta_{p}-\beta_{i}\right)} \\
& \times\left[y_{p i 0}+y_{p i 1}-\frac{\left(1-y_{p i 0}-y_{p i 1}\right)\left\{1-c+\exp \left(\xi_{0 p}\right)\right\}}{c\left\{1+\exp \left(\theta_{p}-\beta_{i}\right)\right\}+\left\{1-c+\exp \left(\xi_{0 p}\right)\right\} \exp \left(\theta_{p}-\beta_{i}\right)}\right] \\
& \times f\left(\theta_{p}, \xi_{0 p}\right) \mathrm{d} \theta_{p} \mathrm{~d} \xi_{0 p}, \\
& I_{4 p i}=\int_{\mathbb{R}^{2}} \frac{A_{p} \delta_{1} \exp \left(\theta_{p}-\beta_{i}\right)}{\left\{1+\exp \left(\xi_{0 p}\right)\right\}\left\{1+\exp \left(\theta_{p}-\beta_{i}\right)\right\}} \\
& \times\left[y_{p i 0}+y_{p i 1}-\frac{\left(1-y_{p i 0}-y_{p i 1}\right)\left\{1-c+\exp \left(\xi_{0 p}\right)\right\}}{c\left\{1+\exp \left(\theta_{p}-\beta_{i}\right)\right\}+\left\{1-c+\exp \left(\xi_{0 p}\right)\right\} \exp \left(\theta_{p}-\beta_{i}\right)}\right] \\
& \times \sum_{j=1}^{I} \frac{j}{I}\left\{y_{p j 0}-y_{p j 1} \exp \left(\xi_{0 p}\right)-\frac{\left(1-y_{p j 0}-y_{p j 1}\right) c \exp \left(\xi_{0 p}\right)}{c\left\{1+\exp \left(\theta_{p}-\beta_{j}\right)\right\}+\left\{1-c+\exp \left(\xi_{0 p}\right)\right\} \exp \left(\theta_{p}-\beta_{j}\right)}\right\} \\
& \times f\left(\theta_{p}, \xi_{0 p}\right) \mathrm{d} \theta_{p} \mathrm{~d} \xi_{0 p}, \\
& I_{5 p i}=\int_{\mathbb{R}^{2}} \frac{A_{p} \delta_{1} \exp \left(\xi_{0 p}+\theta_{p}-\beta_{i}\right)}{\left[c\left\{1+\exp \left(\theta_{p}-\beta_{j}\right)\right\}+\left\{1-c+\exp \left(\xi_{0 p}\right)\right\} \exp \left(\theta_{p}-\beta_{j}\right)\right]^{2}} f\left(\theta_{p}, \xi_{0 p}\right) \mathrm{d} \theta_{p} \mathrm{~d} \xi_{0 p}, \\
& I_{6 p}=\int_{\mathbb{R}^{2}} A_{p} g_{1}\left(\theta_{p}, \xi_{0 p}\right) f\left(\theta_{p}, \xi_{0 p}\right) \mathrm{d} \theta_{p} \mathrm{~d} \xi_{0 p},
\end{aligned}
$$$$
I_{7 p}=\int_{\mathbb{R}^{2}} \frac{A_{p} g_{2}\left(\theta_{p}, \xi_{0 p}\right)}{1+\exp \left(\xi_{0 p}\right)} \sum_{i=1}^{I} \frac{i}{I}\left[y_{p i 0}-y_{p i 1} \exp \left(\xi_{0 p}\right)-\frac{\left(1-y_{p i 0}-y_{p i 1}\right) c \exp \left(\xi_{0 p}\right)}{c\left\{1+\exp \left(\theta_{p}-\beta_{i}\right)\right\}+\left\{1-c+\exp \left(\xi_{0 p}\right)\right\} \exp \left(\theta_{p}-\beta_{i}\right)}\right]
$$$$
\times f\left(\theta_{p}, \xi_{0 p}\right) \mathrm{d} \theta_{p} \mathrm{~d} \xi_{0 p},
$$

$$
I_{8 p}=\int_{\mathbb{R}^{2}} A_{p} g_{3}\left(\theta_{p}, \xi_{0 p}\right) f\left(\theta_{p}, \xi_{0 p}\right) \mathrm{d} \theta_{p} \mathrm{~d} \xi_{0 p}
$$




$$
\begin{aligned}
I_{9 p}= & \int_{\mathbb{R}^{2}} \frac{A_{p} g_{4}\left(\theta_{p}, \xi_{0 p}\right)}{1+\exp \left(\xi_{0 p}\right)} \sum_{i=1}^{I} \frac{i}{I}\left[y_{p i 0}-y_{p i 1} \exp \left(\xi_{0 p}\right)-\frac{\left(1-y_{p i 0}-y_{p i 1}\right) c \exp \left(\xi_{0 p}\right)}{c\left\{1+\exp \left(\theta_{p}-\beta_{i}\right)\right\}+\left\{1-c+\exp \left(\xi_{0 p}\right)\right\} \exp \left(\theta_{p}-\beta_{i}\right)}\right] \\
& \times f\left(\theta_{p}, \xi_{0 p}\right) \mathrm{d} \theta_{p} \mathrm{~d} \xi_{0 p},
\end{aligned}
$$

$$
I_{10 p}=\int_{\mathbb{R}^{2}} A_{p} g_{5}\left(\theta_{p}, \xi_{0 p}\right) f\left(\theta_{p}, \xi_{0 p}\right) \mathrm{d} \theta_{p} \mathrm{~d} \xi_{0 p}
$$

$$
\begin{aligned}
I_{11 p}= & \int_{\mathbb{R}^{2}} \frac{A_{p} g_{6}\left(\theta_{p}, \xi_{0 p}\right)}{1+\exp \left(\xi_{0 p}\right)} \sum_{i=1}^{I} \frac{i}{I}\left[y_{p i 0}-y_{p i 1} \exp \left(\xi_{0 p}\right)-\frac{\left(1-y_{p i 0}-y_{p i 1}\right) c \exp \left(\xi_{0 p}\right)}{c\left\{1+\exp \left(\theta_{p}-\beta_{i}\right)\right\}+\left\{1-c+\exp \left(\xi_{0 p}\right)\right\} \exp \left(\theta_{p}-\beta_{i}\right)}\right] \\
& \times f\left(\theta_{p}, \xi_{0 p}\right) \mathrm{d} \theta_{p} \mathrm{~d} \xi_{0 p},
\end{aligned}
$$

$$
I_{12 p}=\int_{\mathbb{R}^{2}} A_{p} g_{7}\left(\theta_{p}, \xi_{0 p}\right) f\left(\theta_{p}, \xi_{0 p}\right) \mathrm{d} \theta_{p} \mathrm{~d} \xi_{0 p},
$$

$$
I_{13 p}=\int_{\mathbb{R}^{2}} \frac{A_{p} g_{8}\left(\theta_{p}, \xi_{0 p}\right)}{1+\exp \left(\xi_{0 p}\right)} \sum_{i=1}^{I} \frac{i}{I}\left[y_{p i 0}-y_{p i 1} \exp \left(\xi_{0 p}\right)-\frac{\left\{1-y_{p i 0}-y_{p i 1}\right\} c \exp \left(\xi_{0 p}\right)}{c\left\{1+\exp \left(\theta_{p}-\beta_{i}\right)\right\}+\left\{1-c+\exp \left(\xi_{0 p}\right)\right\} \exp \left(\theta_{p}-\beta_{i}\right)}\right]
$$
$\times f\left(\theta_{p}, \xi_{0 p}\right) \mathrm{d} \theta_{p} \mathrm{~d} \xi_{0 p}$,

$$
\begin{aligned}
I_{14 p}= & \int_{\mathbb{R}^{2}} A_{p} \sum_{i=1}^{I}\left[-\frac{y_{p i 1}}{1-c}+\frac{1-y_{p i 0}-y_{p i 1}}{c\left\{1+\exp \left(\theta_{p}-\beta_{i}\right)\right\}+\left\{1-c+\exp \left(\xi_{0}\right)\right\} \exp \left(\theta_{p}-\beta_{i}\right)}\right] \\
& \times f\left(\theta_{p}, \xi_{0 p}\right) \mathrm{d} \theta_{p} \mathrm{~d} \xi_{0 p}, \\
I_{15 p}= & \int_{\mathbb{R}^{2}} \frac{A_{p} \delta_{1}}{1+\exp \left(\xi_{0 p}\right)} \sum_{i=1}^{I}\left[-\frac{y_{p i 1}}{1-c}+\frac{1-y_{p i 0}-y_{p i 1}}{c\left\{1+\exp \left(\theta_{p}-\beta_{i}\right)\right\}+\left\{1-c+\exp \left(\xi_{0}\right)\right\} \exp \left(\theta_{p}-\beta_{i}\right)}\right] \\
& \times \sum_{i=1}^{I} \frac{i}{I}\left[y_{p i 0}-y_{p i 1} \exp \left(\xi_{0 p}\right)-\frac{\left(1-y_{p i 0}-y_{p i 1}\right) c \exp \left(\xi_{0 p}\right)}{c\left\{1+\exp \left(\theta_{p}-\beta_{i}\right)\right\}+\left\{1-c+\exp \left(\xi_{0 p}\right)\right\} \exp \left(\theta_{p}-\beta_{i}\right)}\right] \\
& \times f\left(\theta_{p}, \xi_{0 p}\right) \mathrm{d} \theta_{p} \mathrm{~d} \xi_{0 p},
\end{aligned}
$$

$I_{16 p}=\int_{\mathbb{R}^{2}} A_{p} \delta_{1} \exp \left(\xi_{0 p}\right) \sum_{i=1}^{I} \frac{i}{I} \frac{\left(1-y_{p i 0}-y_{p i 1}\right) \exp \left(\theta_{p}-\beta_{i}\right)}{\left[c\left\{1+\exp \left(\theta_{p}-\beta_{i}\right)\right\}+\left\{1-c+\exp \left(\xi_{0 p}\right)\right\} \exp \left(\theta_{p}-\beta_{i}\right)\right]^{2}} f\left(\theta_{p}, \xi_{0 p}\right) \mathrm{d} \theta_{p} \mathrm{~d} \xi_{0 p}$, with $A_{p}=A_{p}(0)$ and

$$
\begin{aligned}
g_{1}\left(\theta_{p}, \xi_{0 p}\right)= & -\frac{1}{2|\Omega|}\left[\sigma_{\xi_{0}}^{2} \sigma_{\xi_{1}}^{2}-\sigma_{23}^{2}-c_{1}\left(\sigma_{\xi_{0}}^{2} \sigma_{\xi_{1}}^{2}-\sigma_{23}^{2}\right) \theta_{p}^{2}+\left\{\sigma_{\xi_{1}}^{2}-c_{1}\left(\sigma_{\theta}^{2} \sigma_{\xi_{1}}^{2}-\sigma_{13}^{2}\right)\right\}\left(\xi_{0 p}-\mu_{\xi_{0}}\right)^{2}\right. \\
& +\left\{\sigma_{\xi_{0}}^{2}-c_{1}\left(\sigma_{\theta}^{2} \sigma_{\xi_{0}}^{2}-\sigma_{12}^{2}\right)\right\} \delta_{2}-2 c_{1}\left(\sigma_{13} \sigma_{23}-\sigma_{12} \sigma_{\xi_{1}}^{2}\right) \theta_{p}\left(\xi_{0 p}-\mu_{\xi_{0}}\right) \\
& -2\left\{\sigma_{23}+c_{1}\left(\sigma_{12} \sigma_{13}-\sigma_{23} \sigma_{\theta}^{2}\right)\right\}\left(\xi_{0 p}-\mu_{\xi_{0}}\right)\left(\mu-\mu_{\xi_{1}}\right) \\
& \left.-2 c_{1}\left(\sigma_{12} \sigma_{23}-\sigma_{13} \sigma_{\xi_{0}}^{2}\right) \theta_{p}\left(\mu-\mu_{\xi_{1}}\right)\right], \\
g_{2}\left(\theta_{p}, \xi_{0 p}\right)= & -\frac{1}{2|\Omega|}\left(\delta _ { 1 } \left[\sigma_{\xi_{0}}^{2} \sigma_{\xi_{1}}^{2}-\sigma_{23}^{2}-c_{1}\left(\sigma_{\xi_{0}}^{2} \sigma_{\xi_{1}}^{2}-\sigma_{23}^{2}\right) \theta_{p}^{2}+\left\{\sigma_{\xi_{1}}^{2}-c_{1}\left(\sigma_{\theta}^{2} \sigma_{\xi_{1}}^{2}-\sigma_{13}^{2}\right)\right\}\left(\xi_{0 p}-\mu_{\xi_{0}}\right)^{2}\right.\right. \\
& \left.-2 c_{1}\left(\sigma_{13} \sigma_{23}-\sigma_{12} \sigma_{\xi_{1}}^{2}\right) \theta_{p}\left(\xi_{0 p}-\mu_{\xi_{0}}\right)\right]+\left\{\sigma_{\xi_{0}}^{2}-c_{1}\left(\sigma_{\theta}^{2} \sigma_{\xi_{0}}^{2}-\sigma_{12}^{2}\right)\right\} \delta_{4} \\
& \left.-2\left\{\sigma_{23}+c_{1}\left(\sigma_{12} \sigma_{13}-\sigma_{23} \sigma_{\theta}^{2}\right)\right\}\left(\xi_{0 p}-\mu_{\xi_{0}}\right) \delta_{3}-2 c_{1}\left(\sigma_{12} \sigma_{23}-\sigma_{13} \sigma_{\xi_{0}}^{2}\right) \theta_{p} \delta_{3}\right), \\
g_{3}\left(\theta_{p}, \xi_{0 p}\right)= & \frac{1}{|\Omega|}\left\{\left(\sigma_{\theta}^{2} \sigma_{\xi_{1}}^{2}-\sigma_{13}^{2}\right)\left(\xi_{0 p}-\mu_{\xi_{0}}\right)+\left(\sigma_{13} \sigma_{23}-\sigma_{12} \sigma_{\xi_{1}}^{2}\right) \theta_{p}+\left(\sigma_{12} \sigma_{13}-\sigma_{23} \sigma_{\theta}^{2}\right)\left(\mu-\mu_{\xi_{1}}\right)\right\}, \\
g_{4}\left(\theta_{p}, \xi_{0 p}\right)= & \frac{1}{|\Omega|}\left[\delta_{1}\left\{\left(\sigma_{\theta}^{2} \sigma_{\xi_{1}}^{2}-\sigma_{13}^{2}\right)\left(\xi_{0 p}-\mu_{\xi_{0}}\right)+\left(\sigma_{13} \sigma_{23}-\sigma_{12} \sigma_{\xi_{1}}^{2}\right) \theta_{p}\right\}+\left(\sigma_{12} \sigma_{13}-\sigma_{23} \sigma_{\theta}^{2}\right) \delta_{3}\right],
\end{aligned}
$$




$$
\begin{aligned}
& g_{5}\left(\theta_{p}, \xi_{0 p}\right)=-\frac{1}{2|\Omega|}\left[\sigma_{\theta}^{2} \sigma_{\xi_{1}}^{2}-\sigma_{13}^{2}+\left\{\sigma_{\xi_{1}}^{2}-c_{2}\left(\sigma_{\xi_{0}}^{2} \sigma_{\xi_{1}}^{2}-\sigma_{23}^{2}\right)\right\} \theta_{p}^{2}-c_{2}\left(\sigma_{\theta}^{2} \sigma_{\xi_{1}}^{2}-\sigma_{13}^{2}\right)\left(\xi_{0 p}-\mu_{\xi_{0}}\right)^{2}\right. \\
& +\left\{\sigma_{\theta}^{2}-c_{2}\left(\sigma_{\theta}^{2} \sigma_{\xi_{0}}^{2}-\sigma_{12}^{2}\right)\right\} \delta_{2}-2 c_{2}\left(\sigma_{13} \sigma_{23}-\sigma_{12} \sigma_{\xi_{1}}^{2}\right) \theta_{p}\left(\xi_{0 p}-\mu_{\xi_{0}}\right) \\
& \left.-2\left\{\sigma_{13}+c_{2}\left(\sigma_{12} \sigma_{23}-\sigma_{13} \sigma_{\xi_{0}}^{2}\right)\right\} \theta_{p}\left(\mu-\mu_{\xi_{1}}\right)-2 c_{2}\left(\sigma_{12} \sigma_{13}-\sigma_{23} \sigma_{\theta}^{2}\right)\left(\xi_{0 p}-\mu_{\xi_{0}}\right)\left(\mu-\mu_{\xi_{1}}\right)\right] \text {, } \\
& g_{6}\left(\theta_{p}, \xi_{0 p}\right)=-\frac{1}{2|\Omega|}\left(\delta _ { 1 } \left[\sigma_{\theta}^{2} \sigma_{\xi_{1}}^{2}-\sigma_{13}^{2}+\left\{\sigma_{\xi_{1}}^{2}-c_{2}\left(\sigma_{\xi_{0}}^{2} \sigma_{\xi_{1}}^{2}-\sigma_{23}^{2}\right)\right\} \theta_{p}^{2}-c_{2}\left(\sigma_{\theta}^{2} \sigma_{\xi_{1}}^{2}-\sigma_{13}^{2}\right)\left(\xi_{0 p}-\mu_{\xi_{0}}\right)^{2}\right.\right. \\
& \left.-2 c_{2}\left(\sigma_{13} \sigma_{23}-\sigma_{12} \sigma_{\xi_{1}}^{2}\right) \theta_{p}\left(\xi_{0 p}-\mu_{\xi_{0}}\right)\right]+\left\{\sigma_{\theta}^{2}-c_{2}\left(\sigma_{\theta}^{2} \sigma_{\xi_{0}}^{2}-\sigma_{12}^{2}\right)\right\} \delta_{4} \\
& \left.-2\left\{\sigma_{13}+c_{2}\left(\sigma_{12} \sigma_{23}-\sigma_{13} \sigma_{\xi_{0}}^{2}\right)\right\} \theta_{p} \delta_{3}-2 c_{2}\left(\sigma_{12} \sigma_{13}-\sigma_{23} \sigma_{\theta}^{2}\right)\left(\xi_{0 p}-\mu_{\xi_{0}}\right) \delta_{3}\right) \text {, } \\
& g_{7}\left(\theta_{p}, \xi_{0 p}\right)=-\frac{1}{|\Omega|}\left[\sigma_{13} \sigma_{23}-\sigma_{12} \sigma_{\xi_{1}}^{2}-c_{3}\left(\sigma_{\xi_{0}}^{2} \sigma_{\xi_{1}}^{2}-\sigma_{23}^{2}\right) \theta_{p}^{2}-c_{3}\left(\sigma_{\theta}^{2} \sigma_{\xi_{1}}^{2}-\sigma_{13}^{2}\right)\left(\xi_{0 p}-\mu_{\xi_{0}}\right)^{2}\right. \\
& -\left\{\sigma_{12}+c_{3}\left(\sigma_{\theta}^{2} \sigma_{\xi_{0}}^{2}-\sigma_{12}^{2}\right)\right\} \delta_{2}-\left\{\sigma_{\xi_{1}}^{2}+2 c_{3}\left(\sigma_{13} \sigma_{23}-\sigma_{12} \sigma_{\xi_{1}}^{2}\right)\right\} \theta_{p}\left(\xi_{0 p}-\mu_{\xi_{0}}\right) \\
& +\left\{\sigma_{23}-2 c_{3}\left(\sigma_{12} \sigma_{23}-\sigma_{13} \sigma_{\xi_{0}}^{2}\right)\right\} \theta_{p}\left(\mu-\mu_{\xi_{1}}\right) \\
& \left.+\left\{\sigma_{13}-2 c_{3}\left(\sigma_{12} \sigma_{13}-\sigma_{23} \sigma_{\theta}^{2}\right)\right\}\left(\xi_{0 p}-\mu_{\xi_{0}}\right)\left(\mu-\mu_{\xi_{1}}\right)\right] \text {, } \\
& g_{8}\left(\theta_{p}, \xi_{0 p}\right)=-\frac{1}{|\Omega|}\left(\delta _ { 1 } \left[\sigma_{13} \sigma_{23}-\sigma_{12} \sigma_{\xi_{1}}^{2}-c_{3}\left(\sigma_{\xi_{0}}^{2} \sigma_{\xi_{1}}^{2}-\sigma_{23}^{2}\right) \theta_{p}^{2}-c_{3}\left(\sigma_{\theta}^{2} \sigma_{\xi_{1}}^{2}-\sigma_{13}^{2}\right)\left(\xi_{0 p}-\mu_{\xi_{0}}\right)^{2}\right.\right. \\
& \left.-\left\{\sigma_{\xi_{1}}^{2}+2 c_{3}\left(\sigma_{13} \sigma_{23}-\sigma_{12} \sigma_{\xi_{1}}^{2}\right)\right\} \theta_{p}\left(\xi_{0 p}-\mu_{\xi_{0}}\right)\right]-\left\{\sigma_{12}+c_{3}\left(\sigma_{\theta}^{2} \sigma_{\xi_{0}}^{2}-\sigma_{12}^{2}\right)\right\} \delta_{4} \\
& \left.+\left\{\sigma_{23}-2 c_{3}\left(\sigma_{12} \sigma_{23}-\sigma_{13} \sigma_{\xi_{0}}^{2}\right)\right\} \theta_{p} \delta_{3}+\left\{\sigma_{13}-2 c_{3}\left(\sigma_{12} \sigma_{13}-\sigma_{23} \sigma_{\theta}^{2}\right)\right\}\left(\xi_{0 p}-\mu_{\xi_{0}}\right) \delta_{3}\right),
\end{aligned}
$$

and

$$
\begin{gathered}
\delta_{1}=E\left(\xi_{1 p} \mid \theta_{p}, \xi_{0 p}\right) \\
=\exp \left(\mu+\frac{\sigma^{2}}{2}\right), \\
\delta_{2}=E\left[\left\{\ln \left(\xi_{1 p}\right)-\mu_{\xi_{1}}\right\}^{2} \mid \theta_{p}, \xi_{0 p}\right] \\
=\left(\mu-\mu_{\xi_{1}}\right)^{2}+\sigma^{2}, \\
\delta_{3}=E\left[\xi_{1 p}\left\{\ln \left(\xi_{1 p}\right)-\mu_{\xi_{1}}\right\} \mid \theta_{p}, \xi_{0 p}\right] \\
=\delta_{1}\left(\mu-\mu_{\xi_{1}}+\sigma^{2}\right), \\
\delta_{4}=E\left[\xi_{1 p}\left\{\ln \left(\xi_{1 p}\right)-\mu_{\xi_{1}}\right\}^{2} \mid \theta_{p}, \xi_{0 p}\right] \\
=\delta_{1}\left\{\left(\mu-\mu_{\xi_{1}}+\sigma^{2}\right)^{2}+\sigma^{2}\right\}, \\
c_{1}=\frac{\sigma_{\xi_{0}}^{2} \sigma_{\xi_{1}}^{2}-\sigma_{23}^{2}}{|\Omega|}, \\
c_{2}=\frac{\sigma_{\theta}^{2} \sigma_{\xi_{1}}^{2}-\sigma_{13}^{2}}{|\Omega|}, \\
c_{3}=\frac{\sigma_{13} \sigma_{23}-\sigma_{12} \sigma_{\xi_{1}}^{2}}{|\Omega|},
\end{gathered}
$$

where all the above expressions are evaluated at $\hat{\boldsymbol{\theta}}$.

In the case that the random effects $\theta_{p}, \xi_{0 p}$ and $\xi_{1 p}$ are independent, the above expressions reduce to

$$
\left.\frac{\partial^{2} l_{p}\left(\boldsymbol{\theta} \mid \omega_{p}\right)}{\partial \beta_{i} \partial \omega_{p}}\right|_{\omega_{p}=0}=\frac{E\left(\xi_{1 p}\right)}{I_{1 p}}\left\{-\frac{I_{2 p} I_{3 p i}}{I_{1 p}}+I_{4 p i}-\left(1-y_{p i 0}-y_{p i 1}\right) c \frac{i}{I} I_{5 p i}\right\}, \quad i=1, \ldots, I,
$$




$$
\begin{gathered}
\left.\frac{\partial^{2} l_{p}\left(\boldsymbol{\theta} \mid \omega_{p}\right)}{\partial \sigma_{\theta}^{2} \partial \omega_{p}}\right|_{\omega_{p}=0}=\frac{E\left(\xi_{1 p}\right)}{2 I_{1 p} \sigma_{\theta}^{2}}\left(-\frac{I_{2 p} I_{6 p}}{I_{1 p}}+I_{7 p}\right), \\
\left.\frac{\partial^{2} l_{p}\left(\boldsymbol{\theta} \mid \omega_{p}\right)}{\partial \mu_{\xi_{0}} \partial \omega_{p}}\right|_{\omega_{p}=0}=\frac{E\left(\xi_{1 p}\right)}{I_{1 p} \sigma_{\xi_{0}}^{2}}\left(-\frac{I_{2 p} I_{8 p}}{I_{1 p}}+I_{9 p}\right), \\
\left.\frac{\partial^{2} l_{p}\left(\boldsymbol{\theta} \mid \omega_{p}\right)}{\partial \sigma_{\xi_{0}}^{2} \partial \omega_{p}}\right|_{\omega_{p}=0}=\frac{E\left(\xi_{1 p}\right)}{2 I_{1 p} \sigma_{\xi_{0}}^{2}}\left(-\frac{I_{2 p} I_{10 p}}{I_{1 p}}+I_{11 p}\right), \\
\left.\frac{\partial^{2} l_{p}\left(\boldsymbol{\theta} \mid \omega_{p}\right)}{\partial c \partial \omega_{p}}\right|_{\omega_{p}=0}=\frac{E\left(\xi_{1 p}\right)}{I_{1 p}}\left(-\frac{I_{2 p} I_{14 p}}{I_{1 p}}+I_{15 p}-I_{16 p}\right),
\end{gathered}
$$

with $E\left(\xi_{1 p}\right)=\exp \left(\mu_{\xi_{1}}+\sigma_{\xi_{1}}^{2} / 2\right)$ and

$$
I_{1 p}=\int_{\mathbb{R}^{2}} A_{p} f_{1}\left(\theta_{p}\right) f_{2}\left(\xi_{0 p}\right) \mathrm{d} \theta_{p} \mathrm{~d} \xi_{0 p}
$$

$$
\begin{aligned}
I_{2 p}= & \int_{\mathbb{R}^{2}} \frac{A_{p}}{1+\exp \left(\xi_{0 p}\right)} \\
& \times \sum_{i=1}^{I} \frac{i}{I}\left[y_{p i 0}-y_{p i 1} \exp \left(\xi_{0 p}\right)-\frac{\left(1-y_{p i 0}-y_{p i 1}\right) c \exp \left(\xi_{0 p}\right)}{c\left\{1+\exp \left(\theta_{p}-\beta_{i}\right)\right\}+\left\{1-c+\exp \left(\xi_{0 p}\right)\right\} \exp \left(\theta_{p}-\beta_{i}\right)}\right] \\
& \times f_{1}\left(\theta_{p}\right) f_{2}\left(\xi_{0 p}\right) \mathrm{d} \theta_{p} \mathrm{~d} \xi_{0 p}, \\
I_{3 p i}= & \int_{\mathbb{R}^{2}} \frac{A_{p} \exp \left(\theta_{p}-\beta_{i}\right)}{1+\exp \left(\theta_{p}-\beta_{i}\right)} \\
& \times\left[y_{p i 0}+y_{p i 1}-\frac{\left(1-y_{p i 0}-y_{p i 1}\right)\left\{1-c+\exp \left(\xi_{0 p}\right)\right\}}{c\left\{1+\exp \left(\theta_{p}-\beta_{i}\right)\right\}+\left\{1-c+\exp \left(\xi_{0 p}\right)\right\} \exp \left(\theta_{p}-\beta_{i}\right)}\right] \\
& \times f_{1}\left(\theta_{p}\right) f_{2}\left(\xi_{0 p}\right) \mathrm{d} \theta_{p} \mathrm{~d} \xi_{0 p},
\end{aligned}
$$

$$
\begin{aligned}
I_{4 p i}= & \int_{\mathbb{R}^{2}} \frac{A_{p} \exp \left(\theta_{p}-\beta_{i}\right)}{\left\{1+\exp \left(\xi_{0 p}\right)\right\}\left\{1+\exp \left(\theta_{p}-\beta_{i}\right)\right\}} \\
& \times\left[y_{p i 0}+y_{p i 1}-\frac{\left(1-y_{p i 0}-y_{p i 1}\right)\left\{1-c+\exp \left(\xi_{0 p}\right)\right\}}{c\left\{1+\exp \left(\theta_{p}-\beta_{i}\right)\right\}+\left\{1-c+\exp \left(\xi_{0 p}\right)\right\} \exp \left(\theta_{p}-\beta_{i}\right)}\right] \\
& \times \sum_{j=1}^{I} \frac{j}{I}\left[y_{p j 0}-y_{p j 1} \exp \left(\xi_{0 p}\right)-\frac{\left(1-y_{p j 0}-y_{p j 1}\right) c \exp \left(\xi_{0 p}\right)}{c\left\{1+\exp \left(\theta_{p}-\beta_{j}\right)\right\}+\left\{1-c+\exp \left(\xi_{0 p}\right)\right\} \exp \left(\theta_{p}-\beta_{j}\right)}\right] \\
& \times f_{1}\left(\theta_{p}\right) f_{2}\left(\xi_{0 p}\right) \mathrm{d} \theta_{p} \mathrm{~d} \xi_{0 p},
\end{aligned}
$$$$
I_{5 p i}=\int_{\mathbb{R}^{2}} \frac{A_{p} \exp \left(\xi_{0 p}+\theta_{p}-\beta_{i}\right)}{\left[c\left\{1+\exp \left(\theta_{p}-\beta_{j}\right)\right\}+\left\{1-c+\exp \left(\xi_{0 p}\right)\right\} \exp \left(\theta_{p}-\beta_{j}\right)\right]^{2}} f_{1}\left(\theta_{p}\right) f_{2}\left(\xi_{0 p}\right) \mathrm{d} \theta_{p} \mathrm{~d} \xi_{0 p},
$$$$
I_{6 p}=\int_{\mathbb{R}^{2}} A_{p}\left(\frac{\theta_{p}^{2}}{\sigma_{\theta}^{2}}-1\right) f_{1}\left(\theta_{p}\right) f_{2}\left(\xi_{0 p}\right) \mathrm{d} \theta_{p} \mathrm{~d} \xi_{0 p},
$$$$
I_{7 p}=\int_{\mathbb{R}^{2}} \frac{A_{p}}{1+\exp \left(\xi_{0 p}\right)}\left(\frac{\theta_{p}^{2}}{\sigma_{\theta}^{2}}-1\right)
$$$$
\times \sum_{i=1}^{I} \frac{i}{I}\left[y_{p i 0}-y_{p i 1} \exp \left(\xi_{0 p}\right)-\frac{\left(1-y_{p i 0}-y_{p i 1}\right) c \exp \left(\xi_{0 p}\right)}{c\left\{1+\exp \left(\theta_{p}-\beta_{i}\right)\right\}+\left\{1-c+\exp \left(\xi_{0 p}\right)\right\} \exp \left(\theta_{p}-\beta_{i}\right)}\right]
$$$$
\times f_{1}\left(\theta_{p}\right) f_{2}\left(\xi_{0 p}\right) \mathrm{d} \theta_{p} \mathrm{~d} \xi_{0 p},
$$ 


$$
\begin{aligned}
& I_{8 p}=\int_{\mathbb{R}^{2}} A_{p}\left(\xi_{0 p}-\mu_{\xi_{0}}\right) f_{1}\left(\theta_{p}\right) f_{2}\left(\xi_{0 p}\right) \mathrm{d} \theta_{p} \mathrm{~d} \xi_{0 p}, \\
& I_{9 p}=\int_{\mathbb{R}^{2}} \frac{A_{p}\left(\xi_{0 p}-\mu_{\xi_{0}}\right)}{1+\exp \left(\xi_{0 p}\right)} \\
& \times \sum_{i=1}^{I} \frac{i}{I}\left[y_{p i 0}-y_{p i 1} \exp \left(\xi_{0 p}\right)-\frac{\left(1-y_{p i 0}-y_{p i 1}\right) c \exp \left(\xi_{0 p}\right)}{c\left\{1+\exp \left(\theta_{p}-\beta_{i}\right)\right\}+\left\{1-c+\exp \left(\xi_{0 p}\right)\right\} \exp \left(\theta_{p}-\beta_{i}\right)}\right] \\
& \times f_{1}\left(\theta_{p}\right) f_{2}\left(\xi_{0 p}\right) \mathrm{d} \theta_{p} \mathrm{~d} \xi_{0 p}, \\
& I_{10 p}=\int_{\mathbb{R}^{2}} A_{p}\left\{\frac{\left(\xi_{0 p}-\mu_{\xi_{0}}\right)^{2}}{\sigma_{\xi_{0}}^{2}}-1\right\} f_{1}\left(\theta_{p}\right) f_{2}\left(\xi_{0 p}\right) \mathrm{d} \theta_{p} \mathrm{~d} \xi_{0 p}, \\
& I_{11 p}=\int_{\mathbb{R}^{2}} \frac{A_{p}}{1+\exp \left(\xi_{0 p}\right)}\left\{\frac{\left(\xi_{0 p}-\mu_{\xi_{0}}\right)^{2}}{\sigma_{\xi_{0}}^{2}}-1\right\} \\
& \times \sum_{i=1}^{I} \frac{i}{I}\left[y_{p i 0}-y_{p i 1} \exp \left(\xi_{0 p}\right)-\frac{\left(1-y_{p i 0}-y_{p i 1}\right) c \exp \left(\xi_{0 p}\right)}{c\left\{1+\exp \left(\theta_{p}-\beta_{i}\right)\right\}+\left\{1-c+\exp \left(\xi_{0 p}\right)\right\} \exp \left(\theta_{p}-\beta_{i}\right)}\right] \\
& \times f_{1}\left(\theta_{p}\right) f_{2}\left(\xi_{0 p}\right) \mathrm{d} \theta_{p} \mathrm{~d} \xi_{0 p}, \\
& I_{14 p}=\int_{\mathbb{R}^{2}} A_{p} \sum_{i=1}^{I}\left[-\frac{y_{p i 1}}{1-c}+\frac{1-y_{p i 0}-y_{p i 1}}{c\left\{1+\exp \left(\theta_{p}-\beta_{i}\right)\right\}+\left\{1-c+\exp \left(\xi_{0}\right)\right\} \exp \left(\theta_{p}-\beta_{i}\right)}\right] \\
& \times f_{1}\left(\theta_{p}\right) f_{2}\left(\xi_{0 p}\right) \mathrm{d} \theta_{p} \mathrm{~d} \xi_{0 p}, \\
& I_{15 p}=\int_{\mathbb{R}^{2}} \frac{A_{p}}{1+\exp \left(\xi_{0 p}\right)} \\
& \times \sum_{i=1}^{I}\left[-\frac{y_{p i 1}}{1-c}+\frac{1-y_{p i 0}-y_{p i 1}}{c\left\{1+\exp \left(\theta_{p}-\beta_{i}\right)\right\}+\left\{1-c+\exp \left(\xi_{0}\right)\right\} \exp \left(\theta_{p}-\beta_{i}\right)}\right] \\
& \times \sum_{i=1}^{I} \frac{i}{I}\left[y_{p i 0}-y_{p i 1} \exp \left(\xi_{0 p}\right)-\frac{\left(1-y_{p i 0}-y_{p i 1}\right) c \exp \left(\xi_{0 p}\right)}{c\left\{1+\exp \left(\theta_{p}-\beta_{i}\right)\right\}+\left\{1-c+\exp \left(\xi_{0 p}\right)\right\} \exp \left(\theta_{p}-\beta_{i}\right)}\right] \\
& \times f_{1}\left(\theta_{p}\right) f_{2}\left(\xi_{0 p}\right) \mathrm{d} \theta_{p} \mathrm{~d} \xi_{0 p}, \\
& I_{16 p}=\int_{\mathbb{R}^{2}} A_{p} \exp \left(\xi_{0 p}\right) \\
& \times \sum_{i=1}^{I} \frac{i}{I} \frac{\left(1-y_{p i 0}-y_{p i 1}\right) \exp \left(\theta_{p}-\beta_{i}\right)}{\left[c\left\{1+\exp \left(\theta_{p}-\beta_{i}\right)\right\}+\left\{1-c+\exp \left(\xi_{0 p}\right)\right\} \exp \left(\theta_{p}-\beta_{i}\right)\right]^{2}} f_{1}\left(\theta_{p}\right) f_{2}\left(\xi_{0 p}\right) \mathrm{d} \theta_{p} \mathrm{~d} \xi_{0 p},
\end{aligned}
$$

and where all expressions are evaluated at $\hat{\boldsymbol{\theta}}$.

\section{References}

Birnbaum, A. (1968) Some latent trait models and their use in inferring an examinee's ability. In Statistical Theories of Mental Test Scores (eds F. M. Lord and M. R. Novick), pp. 394 479. Reading: Addison-Wesley.

Bolt, D. M., Cohen, A. S. and Wollack, J. A. (2002) Item parameter estimation under conditions of test speededness: application of a mixture Rasch model with ordinal constraints. J. Educ. Measmnt, 39, 331-348.

Chatterjee, S. and Hadi, A. S. (1988) Sensitivity Analysis in Linear Regression. New York: Wiley.

Cook, R. D. (1986) Assessment of local influence (with discussion). J. R. Statist. Soc. B, 48, 133-169.

Douglas, J., Kim, H. R., Habing, B. and Gao, F. (1998) Investigating local dependence with conditional covariance functions. J. Educ. Behav. Statist., 23, 129-151.

Ferrara, S., Huynh, H. and Michaels, H. (1999) Contextual explanations of local dependence in item clusters in a large-scale hands-on science performance assessment. J. Educ. Measmnt, 36, 119-140. 
Goegebeur, Y., DeBoeck, P., Wollack, J. A. and Cohen, A. S. (2005) A speeded item response model with gradual process change. Technical Report. Department of Psychology, Catholic University of Leuven, Leuven.

Hogan, J. W. and Laird, N. M. (1997) Mixture models for the joint distribution of repeated measures and event times. Statist. Med., 16, 239-258.

Lee, G., Kolen, M. J., Frisbie, D. A. and Ankenmann, R. D. (2001) Comparison of dichotomous and polytomous item response models in equating scores from tests composed of testlets. Appl. Psychol. Measmnt, 25, 357-372.

Lesaffre, E. and Verbeke, G. (1998) Local influence in linear mixed models. Biometrics, 54, 570-582.

Levine, M. V. and Drasgow, F. (1988) Optimal appropriateness measurement. Psychometrika, 53, $161-176$.

Littell, R., Milliken, G., Stroup, W., Wolfinger, R. and Schabenberger, O. (2006) SAS for Mixed Models. Cary: SAS Institute.

Little, R. J. A. (1995) Modeling the drop-out mechanism in repeated measures studies. J. Am. Statist. Ass., 90, $1112-1121$.

Little, R. J. A. and Rubin, D. B. (2002) Statistical Analysis with Missing Data, 2nd edn. New York: Wiley.

Meijer, R. R. and Sijtsma, K. (2001) Methodology review: evaluating person fit. Appl. Psychol. Measmnt, 25, $107-135$.

Molenberghs, G. and Verbeke, G. (2005) Models for Discrete Longitudinal Data. New York: Springer.

Numerical Algorithms Group (1993) NAG Fortran Library Manual-Mark 19. Oxford: Numerical Algorithms Group.

Oshima, T. C. (1994) The effect of speededness on parameter estimation in item response theory. J. Educ. Measmnt, 31, 200-219.

Rasch, G. (1960) Probabilistic Models for Some Intelligence and Attainment Tests. Copenhagen: Danish Institute for Educational Research.

Rubin, D. B. (1976) Inference and missing data. Biometrika, 63, 581-592.

San Martín, E., del Pino, G. and De Boeck, P. (2006) IRT models for ability based guessing. Appl. Psychol. Measmnt, 30, 1-21.

Schafer, J. L. (1997) Analysis of Incomplete Multivariate Data. New York: Chapman and Hall.

Sireci, S. G., Thissen, D. and Wainer, H. (1991) On the reliability of testlet-based tests. J. Educ. Measmnt, 28, 237-247.

Thissen, D., Steinberg, L. and Mooney, J. (1989) Trace lines for testlets: a use of multiple-categorical response models. J. Educ. Measmnt, 26, 247-260.

Tuerlinckx, F. and De Boeck, P. (2001) The effect of ignoring item interactions on the estimated discrimination parameters in item response theory. Psychol. Meth., 6, 181-195.

Verbeke, G. and Molenberghs, G. (2000) Linear Mixed Models for Longitudinal Data. New York: Springer.

Verbeke, G., Molenberghs, G., Thijs, H., Lesaffre, E. and Kenward, M. G. (2001) Sensitivity analysis for nonrandom dropout: a local influence approach. Biometrics, 57, 7-14.

Wainer, H. and Thissen, D. (1996) How is reliability related to the quality of test scores?: what is the effect of local dependence on reliability? Educ. Measmnt Iss. Pract., 15, 22-29.

Wollack, J. A. and Cohen, A. S. (2005) A model for simulating speeded test data. Technical Report. University of Wisconsin-Madison, Madison.

Yamamoto, K. and Everson, H. (1997) Modeling the effects of test length and test time on parameter estimation using the hybrid model. In Applications of Latent Trait and Latent Class Models in the Social Sciences (eds J. Rost and R. Langeheine), pp. 89-99. New York: Waxmann.

Yen, W. M. (1984) Effects of local item dependence on the fit and equating performance of the three-parameter logistic model. Appl. Psychol. Measmnt, 8, 125-145.

Yen, W. M. (1993) Scaling performance assessments: strategies for managing local item dependence. J. Educ. Measmnt, 30, 187-213. 